\title{
Design Optimization of Dental Implants Using Additively Manufactured Lattice Materials
}

\author{
by \\ Soraya Mareishi \\ BSc in Mechanical Engineering
}

A thesis submitted to the Faculty of Graduate and Postdoctoral Affairs in partial fulfillment of the requirements for the degree of Master of Applied Science in

Mechanical Engineering

Carleton University

Ottawa, Ontario

(C) 2018, Soraya Mareishi

(C) 2018, Soraya Mareishi 


\begin{abstract}
A dental implant is a biocompatible surgical component placed into the jawbone to support dental prostheses including bridges, crowns, or denture replacements. Currently, dental implants are constructed employing solid materials, coated with biocompatible layers. Since bone is a living tissue that is constantly modified in response to external loading, redistributed or reduced mechanical loading might cause bone resorption, implant loosening or interface failure, all of which have been notable problems for orthopedic implants. To overcome these issues, we propose a new design for the dental implant structure that can simultaneously minimize bone loss and interface failure. Multiscale and multi-objective design optimization of dental implants employing lattice materials was performed by considering lattice structure in implant design. A 3D FEA model of a segment of jawbone and implant was developed. Mechanical properties of the lattice material were determined and applied to the model and finally, the bone loss and interface failure were studied and the implant structure was optimized based on required objective functions. The proposed design was capable of simultaneously reducing the interface failure and bone loss, as well as implant weight. The optimized implant can be manufactured by additive manufacturing. Additive manufacturing technology facilitates the production of complicated shapes and geometries that cannot be produced by conventional manufacturing processes. In general, the proposed method can be applied to other types of implants and prostheses to optimize their structural performance and reduce their weights.
\end{abstract}




\section{Acknowledgements}

I would like to express my sincere gratitude to my supervisors Professor Fred. F Afagh and Professor Mostafa El Sayed for their continuous support of my graduate study, for the many insightful conversations during the development of this work and their helpful comments. I am thankful for their aspiring guidance, advices and constructive criticism during this project.

I would like to thank my parents for their love, support and sacrifices throughout my life. I would also like to thank my dear husband, Mohammad, for providing me with unfailing support and encouragement. This journey would not have been possible without his support and love. Finally, I must express my gratitude to my siblings, my friends and all those people who inspired me every single day of my life to believe in myself and pursue my dreams. 


\section{Table of Contents}

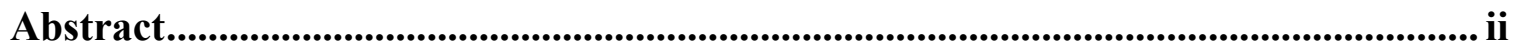
Acknowledgements .......................................................................................................ii

Table of Contents ....................................................................................................... iv

List of Tables ............................................................................................................................... vii

List of Figures................................................................................................................................ viii

List of Acronyms ..........................................................................................................

List of Symbols ................................................................................................................................. xii

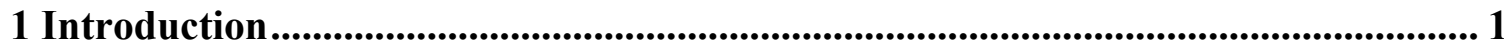

$1.1 \quad$ Background and Motivation ………………………….................................... 1

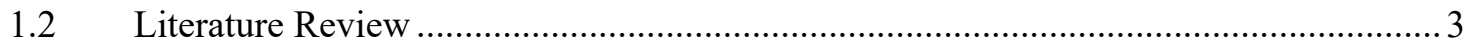

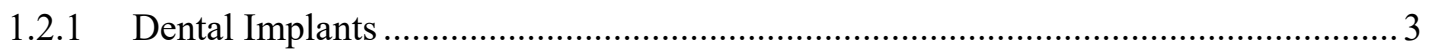

1.2.2 Cellular Solids ...........................................................................................

1.2.3 Design Optimization of Dental Implants .................................................................... 11

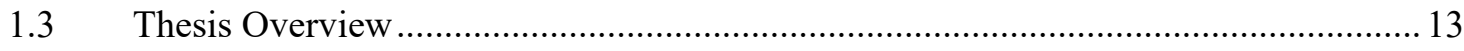

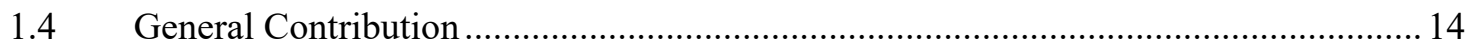

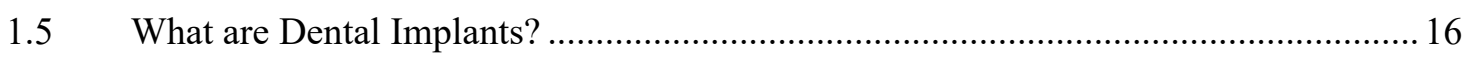

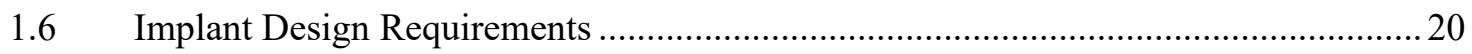

$1.7 \quad$ Failure Mechanisms of Dental Implants ...................................................................2 20

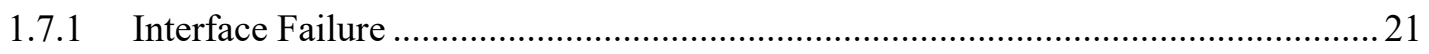

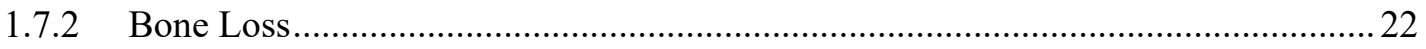

2 Lattice Materials ............................................................................................................. 23

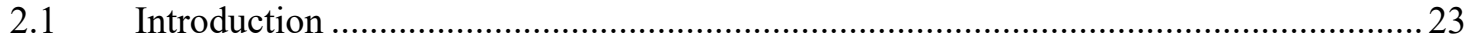




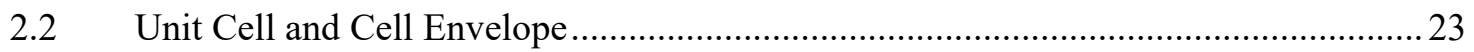

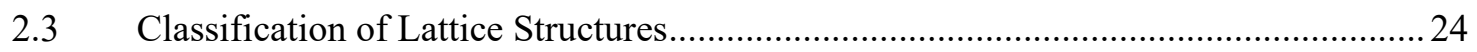

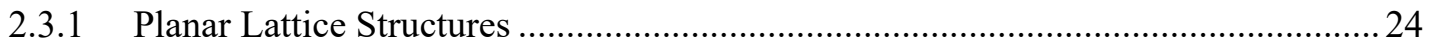

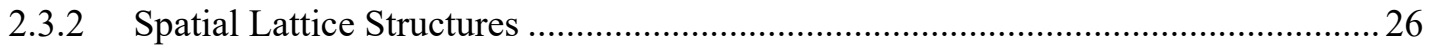

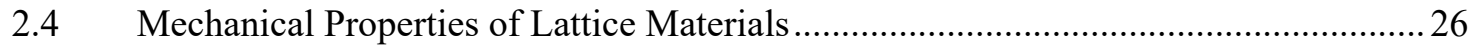

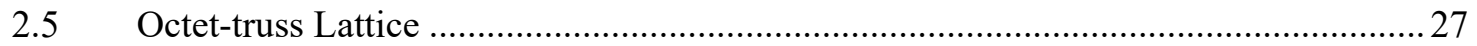

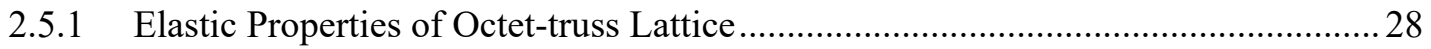

2.5.2 Strength Properties of Octet-truss Lattice: Plastic Yield Strength ...........................29

2.5.3 Strength Properties of Octet-truss Lattice: Elastic Buckling ................................... 30

3 Optimization .............................................................................................................. 32

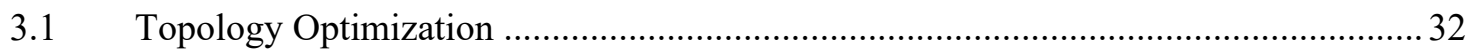

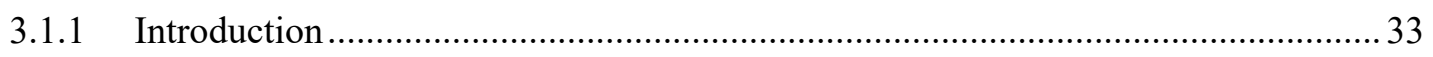

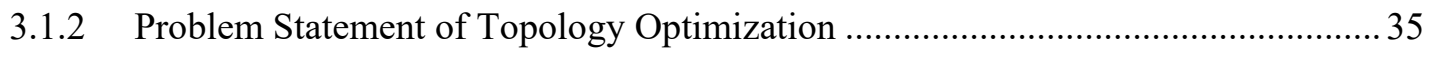

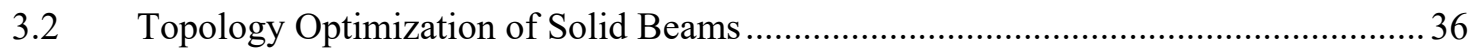

3.2.1 Rectangular Cantilever Solid Beam Topology Optimization................................... 37

3.2.2 Rectangular Simply Supported Beam Topology Optimization............................... 38

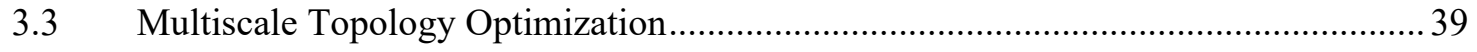

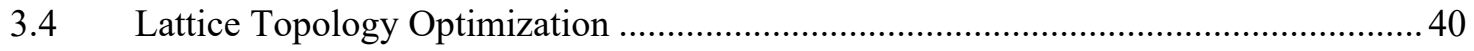

3.4.1 Rectangular Cantilever Beam Lattice Optimization .............................................. 41

3.4.2 Rectangular Simply Supported Beam Lattice Optimization ................................. 43

3.5 Lattice Topology Optimization of Dental Implant with Minimal Compliance ............ 45

4 Multiscale and Multi-Objective Design Optimization of Dental Implant Employing

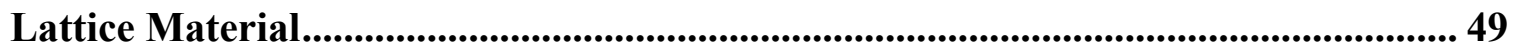

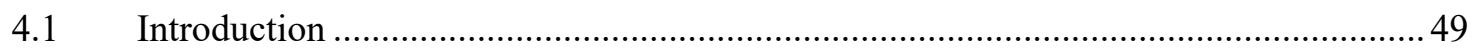

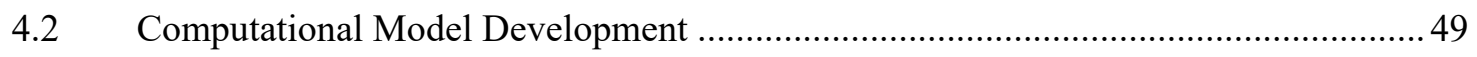




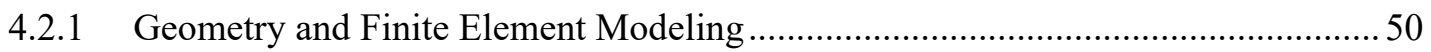

4.2.2 Boundary Conditions and Critical Loading ....................................................... 52

4.3 Effective Mechanical Properties of the Lattice Material ............................................. 53

4.4 Optimization of Implant with Minimum Interface Failure and Bone Loss ...................55

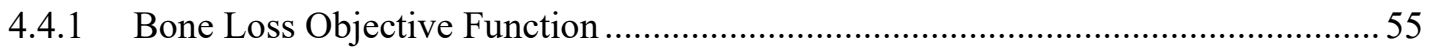

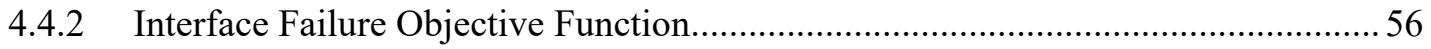

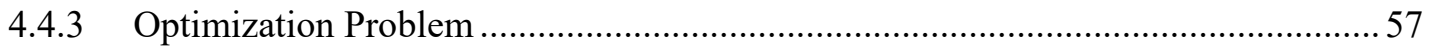

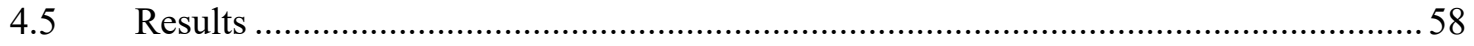

4.5.1 Maximum Von Mises Stress in Lattice Zone ...................................................... 58

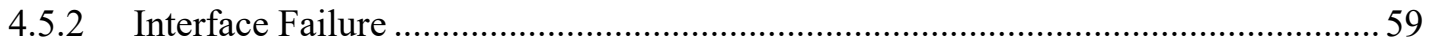

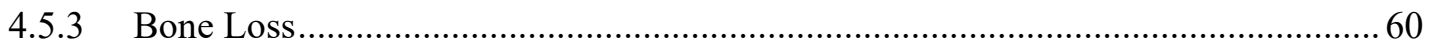

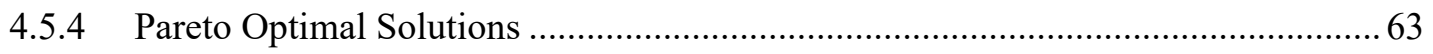

4.5.5 Stress Distribution in Jaw Bone for Solid and Lattice Implants ............................65

4.5.6 Maximum Von Mises Stress in Jaw Bone for Solid and Lattice Implants............... 68

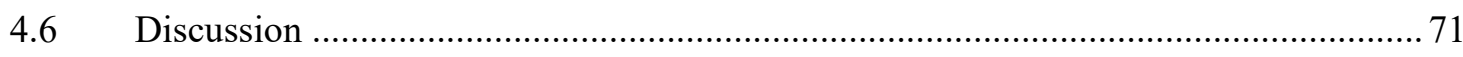

5 Conclusions and Future Work........................................................................................ 73

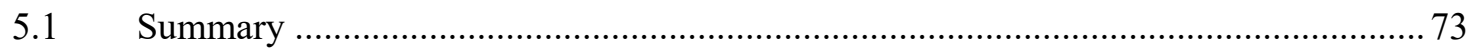

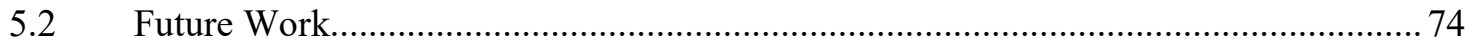




\section{List of Tables}

Table 2.1: Stiffness and strength relation with relative density for lattice materials........ 27

Table 3.1: Summary of mass reduction in topology and multiscale optimization of

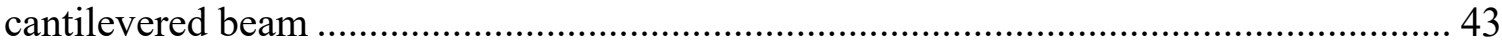

Table 3.2: Summary of mass reduction in topology and multiscale optimization of simply

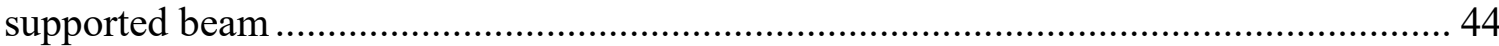

Table 4.1: Mechanical properties of materials used in this study ................................. 51

Table 4.2: Maximum Von Mises stress (MPa) in lattice zone for different relative densities

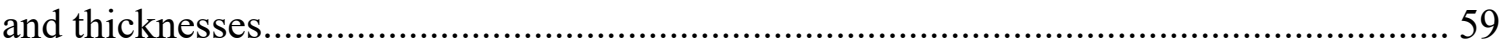

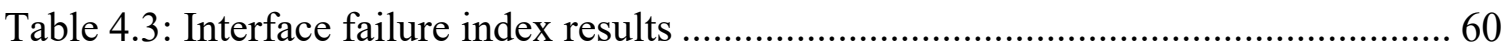

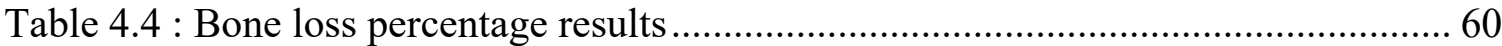




\section{List of Figures}

Figure 1.1: Three-dimensional view of dental implant [1] .......................................... 1

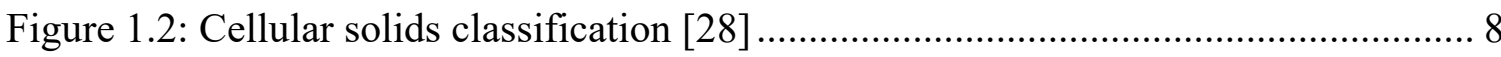

Figure 1.3: The structure of bone, tooth and implant [49] ........................................... 16

Figure 1.4: Implants classification based on design type [51] ...................................... 17

Figure 1.5: Implants classification based on microscopic body design [51] .................. 18

Figure 2.1: Microstructure of cellular materials: (a) Triangular 2D lattice structure; (b) 2D lattice structure with topology of Schlafli symbol [55] .............................................. 24

Figure 2.2: 2D regular planar lattices: (a) Square lattice; (b) Hexagonal lattice; (c)

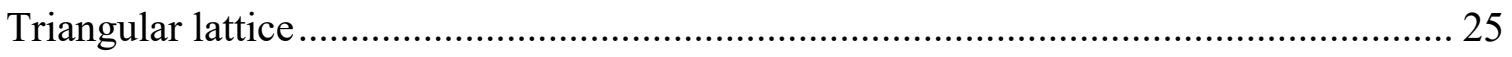

Figure 2.3: (a) Kagome 2D lattice structure; (b) Cell envelop; (c) Unit cell .................... 25

Figure 2.4: 3D polyhedral cells: (a) Tetrahedron; (b) Triangular prism; (c) Square prism; (d) Hegxagonal prism; (e) Octahedron; (f) Rhombic dodecahedron; (g) Dodecaherdon; (h)

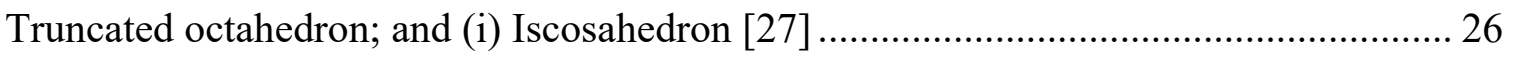

Figure 2.5: Structure of a regular octet-truss lattice unit cell [58] ................................. 28

Figure 3.1: Typical topology optimization problem [61] .......................................... 34

Figure 3.2: Rectangular cantilever beam subjected to a transverse load .......................... 37

Figure 3.3: Topology optimization of a cantilever beam.............................................. 38

Figure 3.4: Simply supported beam subjected to transverse load..................................... 38

Figure 3.5: Topology optimization of a simply supported beam....................................... 39

Figure 3.6: Optimized result for a cantilever plate problem using unpenalized SIMP [72] 
Figure 3.7: Lattice optimization of a cantilever beam (material layout) ....................... 42

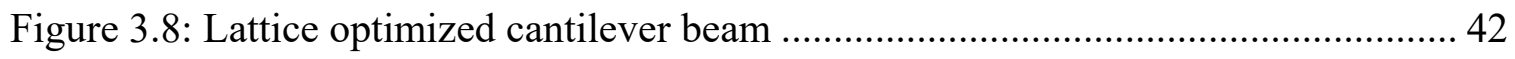

Figure 3.9: Lattice optimization of a simply supported beam (material layout) ............. 43

Figure 3.10: Lattice optimized simply supported beam.......................................... 44

Figure 3.11: Geometry and dimension of bone segment ..................................... 45

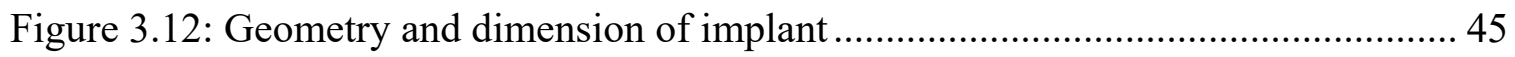

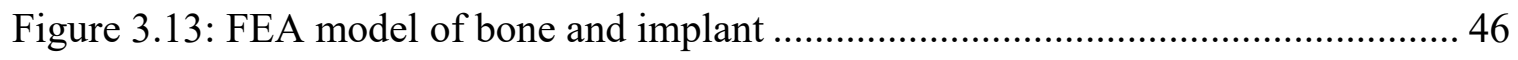

Figure 3.14: FEA model of the bone and implant and loading conditions ..................... 46

Figure 3.15: Material layout of dental implant after optimization............................... 47

Figure 3.16: Final product of implant optimization.............................................. 47

Figure 4.1: Lower mandible (second premolar region is shown in the picture) [78] ....... 50

Figure 4.2: FEA model of bone and implant ...................................................... 51

Figure 4.3: Applied loads and boundary conditions of FEM model. ............................ 53

Figure 4.4: The effect of relative density and implant interface thickness at interface failure

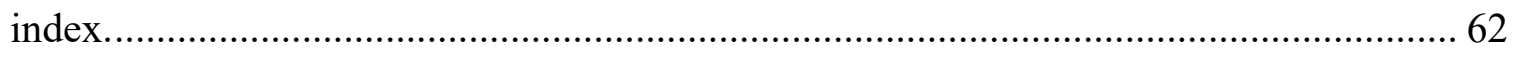

Figure 4.5: The effect of relative density and implant interface thickness at bone loss

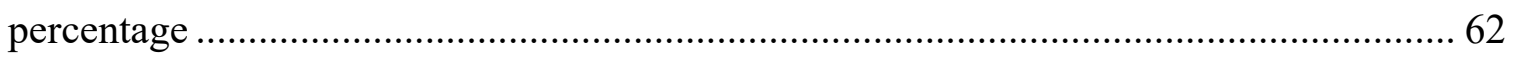

Figure 4.6: Pareto optimal solutions of bone loss and interface failure.......................... 64

Figure 4.7: Pareto frontier solutions of bone loss and interface failure ......................... 65

Figure 4.8: Von Mises stress distribution in cancellous bone in presence of a solid implant

Figure 4.9: Von Mises stress distribution in cortical bone in presence of a solid implant 66 
Figure 4.10: Von Mises stress distribution in cancellous bone in presence of implant typeE

Figure 4.11: Von Mises stress distribution in cortical bone in presence of implant type E 67

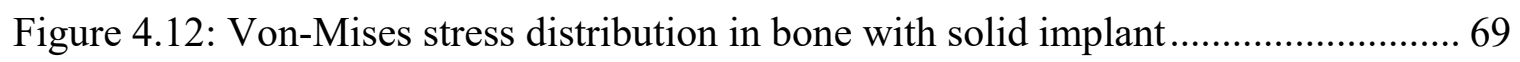

Figure 4.13: Von-Mises stress distribution in bone with lattice implant type (A), ........ 69

Figure 4.14: Von-Mises stress distribution in bone with lattice implant type (C)........... 70

Figure 4.15: Von-Mises stress distribution in bone with lattice implant type (E) ........... 70

Figure 4.16: Implant structure after optimization .............................................. 71 


\section{List of Acronyms}

\begin{tabular}{ll}
\hline Acronyms & Definition \\
\hline AM & Additive manufacturing \\
BCC & Body centered cube \\
BDLM & Bending dominated lattice material \\
BESO & Bi-directional evolutionary structural optimization \\
CAD & Computer aided design \\
FCC & Face centered cube \\
FE & Finite element \\
FEA & Finite element analysis \\
FGM & Functionally graded material \\
GA & Genetic algorithm \\
GSO & Generalized shape optimization \\
LO & Layout optimization \\
SA & Simulated annealing \\
SDLM & Stretching dominated lattice material \\
SIMP & Solid isotropic microstructure with penalization \\
TO & Topology optimization \\
2D & Two-dimensional \\
\hline & Three-dimensional \\
\hline
\end{tabular}




\section{List of Symbols}

\begin{tabular}{|c|c|}
\hline Symbols & Definition \\
\hline$a_{0}$ & Radius of cell element in a lattice unit cell \\
\hline$b$ & Design variable \\
\hline$d$ & Diameter \\
\hline$E_{0}$ & Solid material Young's modulus \\
\hline $\bar{E}_{L a}$ & Relative Young's modulus of lattice \\
\hline$E_{L a}$ & Lattice Young's modulus \\
\hline$F$ & Force \\
\hline$F(b)$ & Interface failure index \\
\hline$f(\sigma)$ & Interface failure probability \\
\hline $\mathrm{f}$ & Force vector \\
\hline$g(s(b))$ & Resorptive function \\
\hline$G_{0}$ & Solid material shear modulus \\
\hline $\bar{G}_{L a}$ & Relative shear modulus of lattice \\
\hline$G_{L a}$ & Lattice shear modulus \\
\hline$K(x)$ & Stiffness matrix \\
\hline$l_{0}$ & Element length in lattice unit cell \\
\hline$L$ & Length \\
\hline$m_{r}$ & Bone loss \\
\hline$M$ & Mass \\
\hline
\end{tabular}


Strain energy density

Reference strain energy

Uniaxial compressive strength

Shear strength

Uniaxial tensile strength

Dead zone value

Thickness

Local strain energy

Displacement vector

Volume

Volume fraction

Width

Lattice Poisson's ratio

Relative density

Lattice density

Relative density of lattice

Solid material density

Normal stress

Solid material yield strength

Relative yield strength of lattice 


$\begin{array}{ll}\sigma_{y L a} & \text { Lattice yield strength } \\ \bar{\sigma}_{L a}^{c r} & \text { Relative critical buckling strength of lattice } \\ \sigma_{L a}^{c r} & \text { Lattice critical buckling strength } \\ \tau & \text { Shear stress } \\ \bar{\tau}_{y L a} & \text { Relative shear strength of lattice } \\ \tau_{y L a} & \text { Lattice shear strength } \\ \tau_{y 0} & \text { Solid material shear strength } \\ \Omega & \\ \tau & \text { Bone-implant interface area }\end{array}$




\section{Introduction}

This chapter starts with the background and motivation of the thesis. Then literature reviews of dental implants, cellular solids and design optimization of dental implants, are provided. Finally, the thesis overview is explained.

\subsection{Background and Motivation}

Development of an ideal substitute for missing teeth has been one of the long-term aims of dentistry. A dental implant is a biocompatible screw-like fixture that is surgically placed into the jawbone to replace the root of the natural tooth. The implant usually acts as an anchor to hold the replacement tooth in place and it is the safest, most functional and efficient method among other techniques for missing tooth replacement. Figure 1.1 provides some details of a typical implant design.

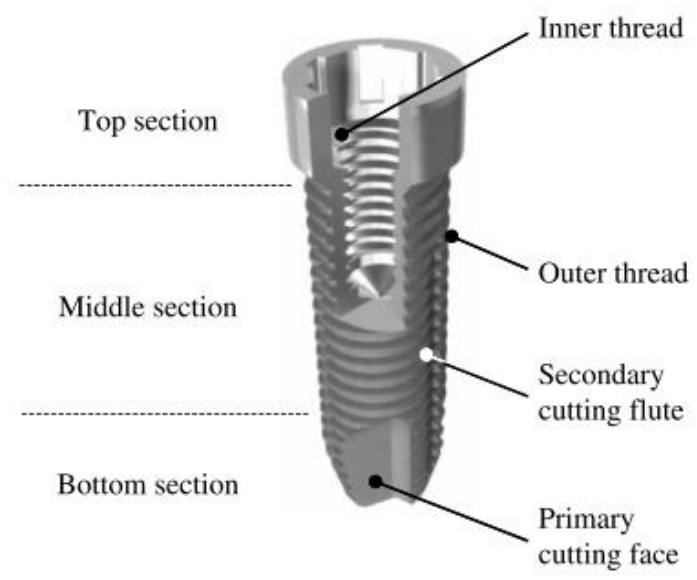

Figure 1.1: Three-dimensional view of dental implant [1] 
The lower part of the implant is inserted into the jaw and is in direct contact with the jawbone. During healing, bone grows around the implant and provides a strong structural support for the implant [2].

The long-term benefits of dental implants include improved appearance, comfort, speech and self-esteem. With the dental implant, the patient can eat more conveniently and the inconvenience of, and at times embarrassment caused by, removable partial and full dentures can be eliminated [3]. Although, the success rate of dental implants is relatively high, occasionally, they fail to remain osseo-integrated and this leads to bone loss and implant instability. A successful osseointegration implant is subject to an appropriate biomechanical environment, which affects the material properties of the implant and prosthesis, implant geometry, surface structure, quality and quantity of the surrounding bone, and nature of the bone-implant interface [4]. Bone loss happens when more bone is resorbed than is formed by the body and it depends on many factors including biocompatibility of the implant, its geometry and surface characteristics, the surgical technique, the restorative treatment and the loading condition [5]. Particularly, it is found that bone loss usually happens because of a mechanical mismatch between the implant and the bone. Current dental implants are often made out of metals like zirconia and titanium alloys. The elastic moduli of these materials are significantly higher than that of bone. For instance, the Young's modulus of bone usually varies between 1-17 GPa, while the Young's modulus of titanium is about $110 \mathrm{GPa}$. As known from solid mechanics, when two different materials are joined, the stiffer structure carries the majority of the load. Once a metal implant is inserted into the bone, the majority of load will be transferred to the implant due to its high stiffness [6]. Therefore, bone will be under-loaded resulting in bone 
loss or deterioration and atrophy, which will increase the bone porosity and make the implant instable as a result of stress shielding. Using porous metals in dental implant design has been considered as one of the solutions to overcome these problems. Porous metals have the ability to allow for bone ingrowth and avoid stress shielding by reducing stiffness of the implant without much loss of its strength. Thus, porous metals are suitable as bone substitute materials in load-bearing applications [7]. In addition, many coating and roughening techniques are used to improve cell and bone-bonding to the implant surface. Several studies have revealed that porous geometry may be a promising alternative to bulk structures for dental implant applications. Cellular structures can provide a suitable biological environment for the host tissue to grow into the pores providing improved early implant stability [8]. So far, numerous studies have been carried out to optimize the structure of the implant, some of which included the use of porous solid structures $[4,9-$ $11]$.

The aim of this thesis is to develop a new dental implant, designed as non-stochastic cellular solids mostly known as lattice materials that can simultaneously reduce the bone loss and implant instability as well as reduce the implant weight.

\subsection{Literature Review}

\subsubsection{Dental Implants}

Worldwide statistics show a high success rate of implantation, in excess of $95 \%$, if the implants are properly designed, manufactured and inserted [1] . Implants are expected to be functioning for a life-long period. This is justified by the fact that the survival rate of the implant at 15 years is as high as $90 \%$ if proper and professional care is taken [12]. The 
success of a dental implant depends on a variety of factors including the design of the abutment and the technique by which the abutment screw is placed into the implant. Major causes of implant failure are due to insufficient biomechanical bonding between the implant and the surrounding jawbone as well as implant tooth fixtures or abutment failure. Insufficient biomechanical bonding might be initiated by, firstly, insufficient osseointegration because of the jawbone not accepting the implant. Secondly, after insertion, the implant might fail due to the lack of hygiene or overload. A solution would be to insert a new implant after the jawbone has restored itself. Other implant failures are due to inaccurate design of the tooth fixture leading to overload of the abutment screw and eventually micro fractures within the implant [1].

Another key factor for the success or failure of a dental implant is the manner in which stresses are transferred to the surrounding bone. Load transfer from implants to surrounding bone depends on the type of loading, the bone-implant interface, the length and diameter of the implants, the shape and characteristics of the implant surface, the prosthesis type, and the quantity and quality of the surrounding bone [13].

Dental implants must withstand relatively large forces sometimes more than $200 \mathrm{~N}$ and resulting large moments in function [14]. Brunski [14] studied in-vivo bone response to biomechanical loading at the bone/dental implant interface. He investigated theoretical models and experimental data for implant loading as an aid to designing an implant.

Analyzing force transfer at the bone-implant interface is also an essential step in the overall analysis of loading, which determines the success or failure of an implant. It has long been recognized that both implant and bone should be stressed within a certain range for physiologic homeostasis. Overloading can cause bone resorption or fatigue failure of the 
implant, whereas underloading of the bone may lead to disuse atrophy and subsequent bone loss [15]. In 1993 Clift, Fisher and Watson [16] analyzed the stress and strain distributions in the bone surrounding a dental implant having a fully bonded interface to the bone. Their design showed $50 \%$ reduction in stress concentration which would help to reduce fatigue failure and bone resorption in this area under lateral loading. Vidyasagar and Apse [17] reviewed the literature on aspects of implant design on the initial implant stability and bone responses to these factors and identified the role of surface roughness on the initial implant stability.

A comprehensive literature review on the topic of dental implant materials was published by Osman and Swain [18]. They focused on conventional titanium and zirconia implants. In their work, the implant materials are categorized according to their chemical composition or the biological responses to implants that are fabricated from metals, ceramics or polymers. Recently, an increasing amount of research has focused on the biological and mechanical behavior of highly porous structures of metallic biomaterials, as implant materials for dental implants. Particularly, pure titanium and its alloys are typically used due to their outstanding mechanical and biological properties. However, these materials have high stiffness (Young's modulus) in comparison to that of the host bone, which necessitates careful implant design to ensure appropriate distribution of stresses to the adjoining bone, to avoid stress-shielding or overloading, both of which lead to bone resorption [8].

Several attempts have been undertaken to develop biomaterials with mechanical properties well-suited to bone tissue. Most of these studies have aimed at optimizing the important features of interactions between the implant surface and bone tissue. Modifying the implant 
surface can also improve the implant to bone interaction, for instance, plasma spraying have been used with different powder particles such as titanium oxide to coat dental implants [8]. Since bone is a living tissue which is continuously modified by the bone cells in response to external signals, reduced mechanical loading leads to resorption of bone, implant loosening and ultimately failure, which has particularly been a problem for orthopedic implants in the past [19]. Numerous studies have been done to manipulate the mechanical and topographical properties of titanium implants. In many studies, micro and nano porous titanium has been proposed as a promising alternative to solid structures for biomedical and dental implant applications. Porous metals and metallic foams have combinations of properties that cannot be obtained with dense polymers, metals and ceramics or polymer and ceramic foams. For example, the mechanical strength, stiffness and energy absorption of porous metallic foams are much higher than those of dense polymer foams [20]. Various fabrication methods have been used to fabricate porous titanium for different medical implant purposes. However, the size, shape, percentage and distribution of pores were variable and need further optimization [21].

$\mathrm{Li}$ and et al. [22] have studied the feasibility and evaluated the compressive properties of Ti6A14V implants with controlled porosity via electron beam melting process. They found that the compressive yield strength of the Ti6Al4V implants with the porosity of around $51 \%$ is higher than that of human cortical bone. The Young's modulus of these implants is similar to that of cortical bone. The porosities and mechanical properties of porous Ti6A14V implants can be adjusted by changing porous structures, such as strut and pore sizes. Grunsven [23] used different strut thicknesses to produce a diamond lattice structure with graded porosity. He found that the mechanical properties that could be achieved could 
be relevant for orthopedic implants. Ahmadi et al. [24] presented new analytical solutions and closed-form relationships for predicting the elastic modulus, Poisson's ratio, critical buckling load, and yield (plateau) stress of cellular structures made of the diamond lattice unit cell. They compared their results with experimental observations. According to their findings, there was a good agreement between the analytical predictions and experimental observations, whereas the prediction from finite element models were less likely to be accurate when compared with the experimental observations. Otsuki et al. [25] examined sintered porous titanium implants with different levels of porosity ( $50 \%$ and $70 \%)$ and pore sizes $(250-500 \mu \mathrm{m})$ and $(500-1500 \mu \mathrm{m})$. Their results indicated that $500-1500 \mu \mathrm{m}$ was the optimal pore size for bone tissue ingrowth at both levels of porosity. In general, the mechanical and biological performance of the porous metal structure is governed by the combined effects of characteristic features of the porosity such as pore shape, size, distribution and their interconnectivity, as well as the base metal from which the porous structure is made. Therefore, the pore geometry needs to be optimized for each specific case to achieve a suitable environment for the surrounding bone tissue. Several approaches have been proposed to overcome these drawbacks by creating porous biomaterials as a substitute for the classical solid structure. Cellular structures can provide a suitable biological environment for the host tissue to grow into the pores [26].

\subsubsection{Cellular Solids}

Cellular solids are porous medium formed as a combination of solid material and enclosed voids. They are made up of interconnected network of solid struts or plates which form the edges and faces of cells [27]. They are mainly divided into two groups as shown in Figure

\section{2 .}




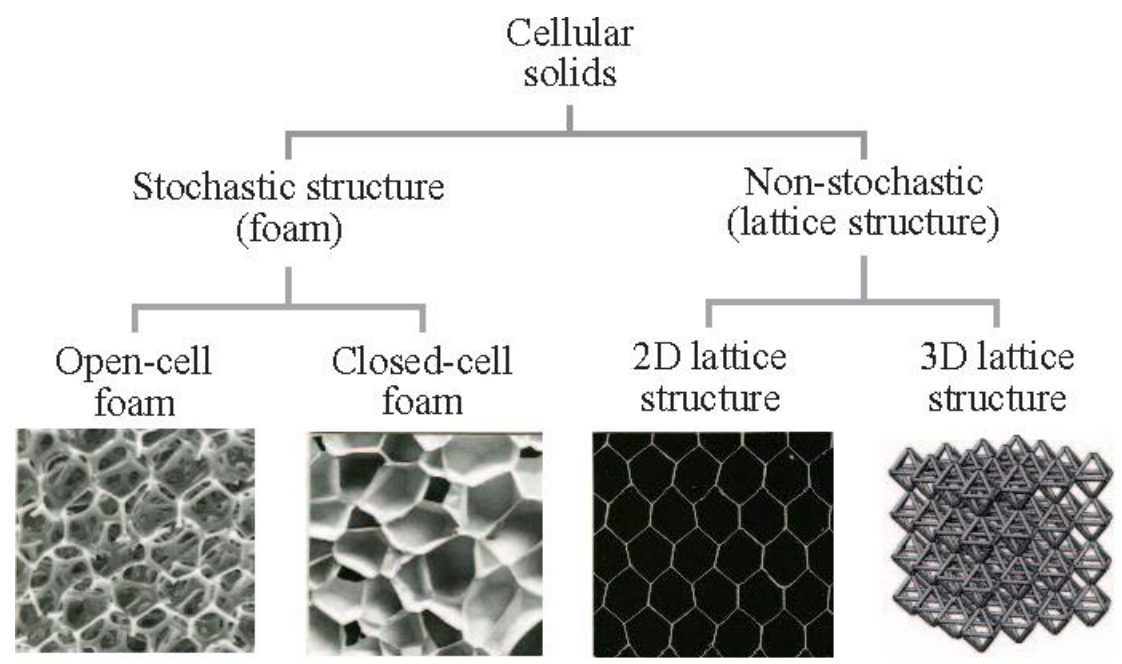

Figure 1.2: Cellular solids classification [28]

Stochastic structures or foams are those type of cellular solids with pore sizes and shapes that are not uniform and they are created randomly, while the non-stochastic structures mainly called lattice materials and honeycombs are built with a periodic uniform shape and size unit cells. These structures are made up of a regular repeating array of simple structural elements. They have certain attractive features, such as the fact that the properties can, in principle, be predicted to greater accuracy than when the pores are randomly located, though they have formerly been difficult to produce in anything other than elementary forms and small sizes, barring certain structures such as honeycombs [26].

Cellular periodic structures or lattice materials are generated by tessellating a unit cell which forms the material representative volume element. The main purpose of creating such materials is reducing the weight, increasing the efficiency by building stiff, strong and load-bearing structures and using as little material as possible. They possess many superior properties to solid material and conventional structures. They are able to integrate more than one function into a physical part, which makes them attractive to a wide range of 
applications. While lattice structures possess complicated geometries, they can be fabricated using additive manufacturing technology using different techniques including the buildup of the material layer-by-layer directly from a computer-aided design (CAD) model, rather than the conventional subtraction manufacturing which requires complicated procedures [28]. At the macroscopic level, lattice materials can be viewed as a material with homogenized properties while the unit cell structure is of dimensions orders of magnitudes lower than those of of the macroscopic structural component. Therfore they can be considered as a homogenized material with their own unique effective properties comparable to solid materials.

To determine the mechanical properties of cellular materials several analytical, numerical and experimental approaches have been studied. Wang and McDowell [22, 23] assumed the cell struts behave like Euler-Bernoulli beams. They examined the individual cell topology and determined the mechanical properties of the unit cell by solving deformation and equilibrium periodic boundary conditions. Cheng et al. [31] used asymptotic homogenization AH to predict the mechanical behavior of periodic materials. Arabnejad and Pasini [32] also studied $\mathrm{AH}$ as a benchmark to test the accuracy of alternative schemes of homogenization applied to lattice materials. They applied AH to determine the effective elastic moduli and yield strength of six lattice topologies for a whole range of relative densities. Hutchinson and Fleck [33] formulated the microscopic nodal deformations of a lattice in terms of the macroscopic strain field in order to derive material macroscopic stiffness properties. A matrix-based procedure to characterize the specific stiffness properties of 2D lattice materials with any arbitrary cell topology was presented by Elsayed and Pasini [34]. They also studied the multiscale structural design of columns made of 
regular octet-truss lattice material and showed that by shaping the cell element crosssections, the lattice material buckling resistance can increase until it equals the cell element yield strength [35]. The mechanical properties of the lattice materials have been studied by Huang and et al.[36]. They showed these properties have obvious dependence on the structural parameters including the inclination angle of the struts with the bottom plane, struts length and strut diameter, etc. Moreover, it was found that the deformation mode of the lattice materials is different if the inclination angle is changed. Messner [37] developed an optimally parameterized, homogenized material model for the periodic structure of lattice materials and also provided an optimization method capable of producing optimal mesostructures. Mancusi and et al. [38] studied the dynamic analysis of two-dimensional (2D) periodic material structures and detected the influence of the characteristics of the lattice at the local scale on the global dynamic behavior. A computational homogenization approach to derive a nonlinear constitutive model for lattice materials was presented by Vigliotti and et al. [39]. The model has been used for the analysis of the hexagonal and the triangulated lattices subjected to large strains. Liu and Zhang [40] developed a uniform multiscale computational method for 2D static and dynamic analyses of lattice truss materials in elasticity based on the extended multiscale finite element method. Vigliotti and Pasini [41] studied the stiffness and strength of lattices with multiple hierarchical levels. They examined two-dimensional and three-dimensional lattices with up to three levels of structural hierarchy (the relative density of the lattice unit cell varies in each level) and they obtained the properties of selected hierarchical lattices via a multiscale approach applied iteratively at each hierarchical level. They showed that by nesting multiple levels 
of lattice hierarchies, and by varying the relative density at each level, the property design space of the solid material can be expanded to reach unexplored areas of the material charts.

One of the most obvious differences between the lattice materials and other cellular materials is that the structure of lattice materials is designable but that of the latter is basically not. This makes the properties of lattice materials not only exactly controllable but optimizable in a wide range. This feature is highly desirable and very important for practical applications, and thus lattice materials have attracted more and more attention of research and industrial circles $[20,27]$. The lattice materials and their mechanical properties will be discussed in Chapter 3 .

\subsubsection{Design Optimization of Dental Implants}

Biomechanical optimization is an important objective in the design of dental implants. Increasingly, it is acknowledged that bone resorption around implants may cause problems. One solution consists in reducing the implant stiffness to match the surrounding bone stiffness by developing optimization methods which can minimize the probability of interface failure, while limiting the amount of bone resorbed [43]. Geng et al. [4] studied the important parameters for a model-based design optimization method through comparison of different biomechanical environments and various dental implants. They found that an oblique load, excellent cortical bone property, and minimal support constraints are critical parameters affecting the stress distribution in the bone-implant model. Therefore, these parameters were important for implant design optimization. Deriving alternative implant shapes which could minimize the stress concentration at the shoulder level of the implant was studied by Shi et al. [44]. They used a topological shape optimization technique in conjunction with the finite element (FE) method to optimize the 
shape of a dental implant under loads. Their design increased the success of dental implants due to the reduced stress concentration at the bone-implant interface. Chahine et al. [9] utilized an analogues approach with FEA, to evaluate the biomechanics of a customized dental implant design built by electron beam melting. They studied three different dental implants in different locations of the jaw bone and by using a deterministic optimization technique in the FEA, they found that the elasticity of the modified layer on implant surface can be selected in a manner to minimize stress shielding. An optimization of the osteoplant dental implant system, with the use of FEA and genetic algorithms was discussed by Lodygowski et al. [45]. They incorporated a FE solver into a genetic optimization procedure and optimized the shape of the dental implant based on FE analyses. The design variables in their work were the geometrical parameters including the height and the diameter of the implant. Design optimization of functionally graded dental implant for bone remodeling was studied by Lin et al. [46]. They developed an optimal design of a dental implant fabricated from functionally graded material (FGM) for promoting a long-term success and showed that a better performance in bone turnover can be achieved by lowering the FGM material gradient. In 2012, Arabnejad-Khanoki and Pasini [47] studied the multiscale design and multi-objective optimization of hip implants with functionally graded cellular material. They used a methodology based on multiscale mechanics and design optimization to synthesize a graded cellular implant that can minimize concurrently bone resorption and implant interface failure. Their results have shown that the optimized cellular implant exhibits a reduction of $76 \%$ in bone resorption and $50 \%$ in interface stress, compared to a fully dense titanium implant. Finite element analysis of the dental implant was studied by Chang et al.[48]. They created three-dimensional FE models by using 
commercial software ANSYS 11.0. Their final design of a new implant was shaped by topology optimization which estimated that the weight of the new implant could be reduced by $17.9 \%$ of the traditional one and the biomechanical performances, such as the stress of the implant, stress of the implant-bone complex, lower displacement, and greater stiffness were similar to those of the traditional implant. Sadollah and Bahreininejad [10] used the concept of functionally graded material to design a FGM dental implant. They used metaheuristic algorithms such as genetic algorithms (GAs) and simulated annealing (SA) to optimize the FGM gradient pattern for desirable on-going bone turnover outcome and mechanical responses.

In the current work, design optimization of dental implant using additive manufactured lattice materials was studied. Topology optimization was used to perform mass minimization and then multiscale and multi-objective design optimization was performed to study the effect of employing lattice material in the structuring of the implant on two important failure functions, namely, interface failure and bone loss. In order to design the required lattice structure, different lattice topologies were studied to determine the optimal implant design.

\subsection{Thesis Overview}

The thesis is organized in five chapters. After this introduction, different types of dental implants and their classification, design requirements and implants failure mechanisms are discussed in Chapter 2. Lattice materials and their mechanical properties are presented in Chapter 3. In Chapter 4, topology optimization and multiscale design optimization are discussed and some of the available useful tools to perform optimization for any kind of 
structure are presented. Also lattice optimization is introduced as an alternative method for solid topology optimization in order to create lighter and stiffer structures.

In Chapter 5, a FEA of the dental implant is presented to evaluate the new proposed implant design. It is assumed that dental implant is made out of a solid core surrounded by an optimized cylindrical part made of lattice material in order to minimize the interface failure and bone loss of surrounding bone. The effective mechanical properties of lattice material with octet-truss topology was adopted and used as the input for the FEA model. The optimization problem is also discussed in Chapter 5 where multiscale and multi-objective design optimization is conducted to determine the optimum interface thickness and lattice microscopic properties in order to minimize the objective functions of the interface failure and bone loss subject to a set of design constraints. Finally, in Chapter 6, a summary of the thesis and future work are discussed.

\subsection{General Contribution}

In this work, design and optimization of dental implant employing lattice material in implant structure was mainly studied. First, a thorough literature review of existing literature about dental implant optimization was performed. Then lattice topology optimization was introduced as one of the possible tools to reduce the implant weight. A multiscale and multiobjective optimization methodology was developed to evaluate the effect of lattice material in implant interface failure and bone loss and the optimal designs were discussed. Finally, it was concluded by using lattice material in implant structure, the objective functions can be minimized. The optimal designs were studied again for stress 
analysis to confirm the change of stresses applied to the bone. Finally, the 3D model of optimized dental implant was generated and weight reductions were found. 


\section{Dental Implants}

In this chapter, a brief explanation of dental implants and their classifications are presented. Then, implant design requirements and failure mechanisms are discussed.

\subsection{What are Dental Implants?}

Dental implants are artificial tooth replacements that are surgically inserted into the jawbone to replace missing or damaged teeth. They are the safest, most functional and efficient way among the techniques for missing tooth replacement applied by dentists [2]. Figure 1.3 represents the structure of the bone and a dental implant as well as the real tooth that will be replaced by the whole implant. As it is shown, the jaw bone is made out of a porous part which is called cancellous bone and a denser part called cortical bone, which surrounds the cancellous. The implant is a screw that serves as a root for the new tooth. It is inserted into the jawbone and is usually made of high-quality metal such as titanium.

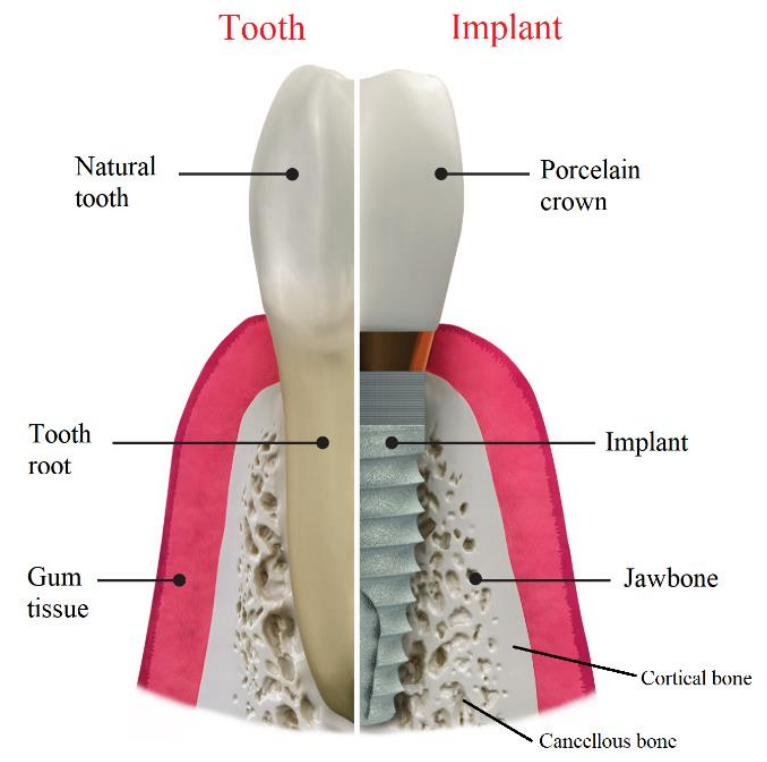

Figure 1.3: The structure of bone, tooth and implant [49] 
Dental implants can be classified based on their shape, design of their microscopic body, type of their surface topology or materials used in their manufacturing [50]. Below, a brief discussion on each of these categories is presented:

\section{Implant design}

As shown in Figure 1.4 implants can be categorized based on their design type, which include endosteal, subperiosteal, transosteal and intramucosal implants.

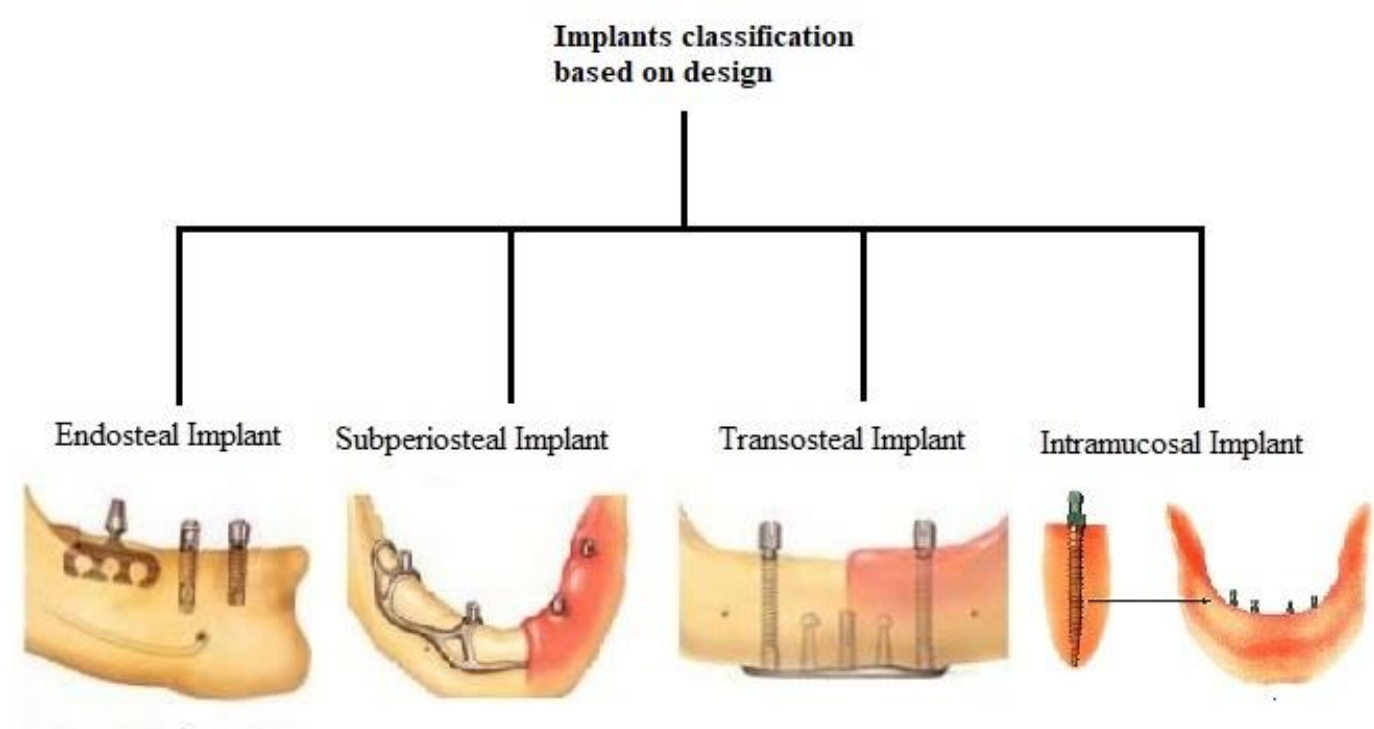

Figure 1.4: Implants classification based on design type [51]

- Endosteal implant: This implant is made up of metallic, ceramic or polymeric material and is available in the form of blade, screw or pin. It is directly placed into the jawbone and is the most common implant used in dentistry.

- Subperiosteal implant: This implant is made out of a metal framework and placed under jaw bone. It is mostly used to cover a large surface area, such as a completely edentulous mandible where there has been a severe amount of bone resorption and not enough bone to support endosteal implants. 
- Transosteal implant: This type of implant is a combination of both the endosteal and those of subperiosteal. The implant penetrates the two cortical plates and it is placed through the chin bone until it gets exposed into the mouth.

- Intramucosal implant: This implant is a mushroom-shaped titanium projection which is used to fix teeth on the upper jaw.

\section{Design of microscopic body}

Dental implants can be classified based on their microscopic body design into cylinder, thread, plateau, perforated, solid and hallow implants as shown in Figure 1.5.

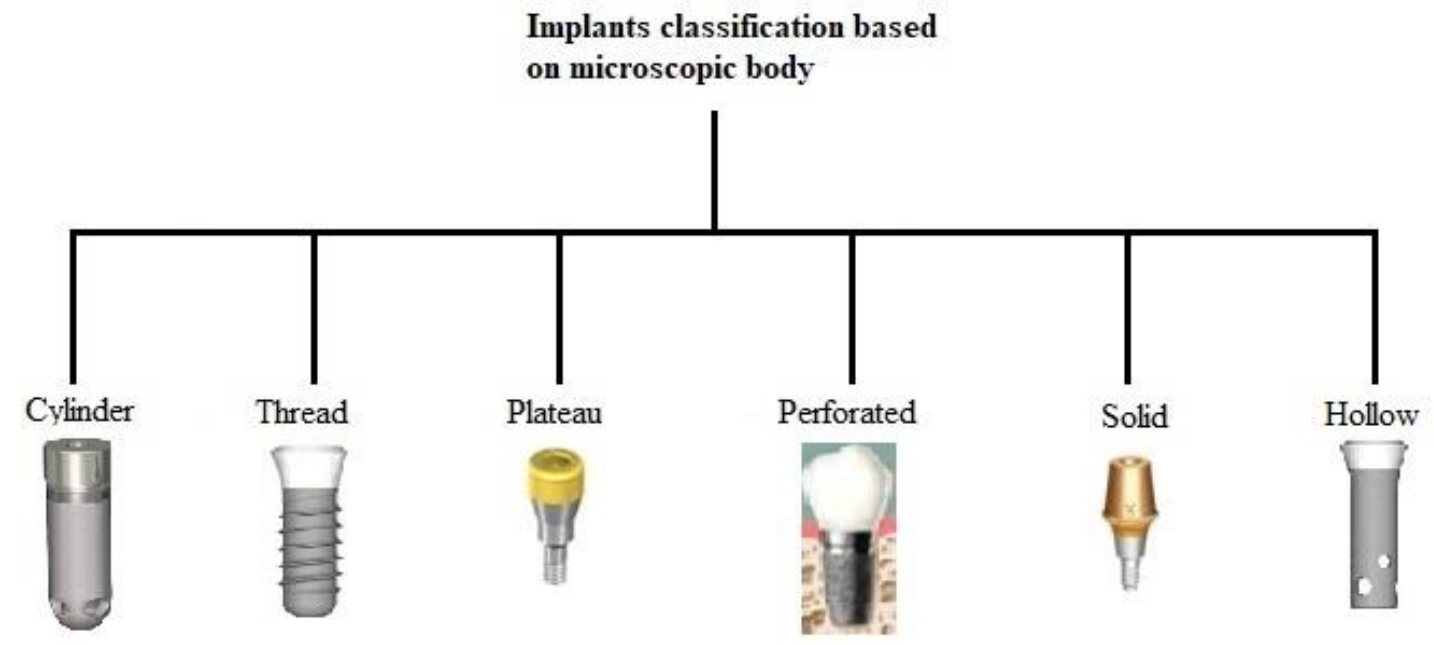

Figure 1.5: Implants classification based on microscopic body design [51]

- Cylinder dental implant: These implants are made in cylindrical shape with a small surface area. They have less compressive force at the bone crest vs tapered (thread) form implants [52]. They also produce a more uniform stress profile with less localized stress concentration in comparison to other implants [2]. 
- Thread dental implant: In this type, the surface of implant is threaded to maximize the surface area of the implant. This results in distribution of forces over greater area. They are currently preferred due to their threads engaging in the body walls which allows for good primary stability as the threads increase the surface area in contact with bone [52].

- Plateau dental implant: This implant has a sloping shoulder and is plateau in shape. It has maximum strength between the bone and the implant surface area.

- Perforated dental implant: These implants are perforated with a mixture of cellulose acetate and are supported by the perforated metallic sheet layer.

- Solid dental implant: This implant has a circular cross section without a vent or hollow in the body

- Hollow dental implant: This one is hollow in the apical portion and perforations are made on the sides of the implant.

\section{Surface of the implant}

Surface of the implant is categorized as smooth, machined, textured and coated. A smooth surface is used to prevent microbial plaque retention. Machined surface is good to provide support to the bone. To increase the roughness between bone and implant, a textured surface can be selected, and coated surface implant which is usually coated with titanium or biocompatible materials can be used to provide a good adjustment between bone and implant.

\section{Materials}

Implants can be classified based on the type of material used in their design, which includes metallic, ceramic, polymer and carbon compounds. The material type can be selected based 
on the required mechanical properties as well as biocompatibility. It should have mechanical properties like tensile strength and Young's modulus that can endure the applied loads and be well tolerated without inducing complications that may occur due to biological or chemical reactions with toxic effects in an environment involving body fluids [2]. The best choice to fulfill the required properties has been found to be titanium. Titanium implants are one of the most common implants being used by dentists. They are biocompatible, corrosion resistant and are preferred alternative to dental dentures since they are more stable, require low maintenance and last for a long time. Titanium has a high tensile strength and a lower Young's modulus compared to other metals. Therefore, the elastic modulus of titanium is closer to the value for bone and it makes it a better choice for implant design.

\subsection{Implant Design Requirements}

In designing an implant, there are several factors that need to be considered. Since bone is a live tissue and implant is in contact with the bone, it is necessary to use materials that are biocompatible. The surface of the implant needs to be high wear and corrosion resistant. The geometric design of implant in terms of manufacturability and simplicity of implantation should also be considered. Ultimate loads applied to dental implants need to be determined.

\subsection{Failure Mechanisms of Dental Implants}

Dental implant failure can happen for many different reasons. No matter how durable or long-lasting they are, they can get affected due to negligence, poor dental care, or external factors. Failure of implants is associated with the failure of the jawbone to merge properly 
together with the implant. An implant is considered to be a failure when it is loose, falls out, or shows signs of bone loss. Many factors can cause this to happen, including: insufficient bone density, incorrect positioning, damage to surrounding tissues, overloading, or fractured implants. One of the main factors is the mismatch of stiffness between the bone and implant causing a non-uniform force transition at the bone-implant interface.

\subsubsection{Interface Failure}

Dental implant interaction with the bone happens through their common interface. While the implant is an immobile structure, bone is a living tissue that can react to mechanical forces. In evaluation of the long-term success of a dental implant, the reliability and stability of the implant-bone interface plays a major role. A key factor to assess the success or failure of a dental implant is the manner in which stresses are transferred to the surrounding bone. Since the implant is usually stiffer than bone, the loads applied to the implant cannot completely be transferred to the bone. This non-uniform stress transition at the bone-implant interface causes interface failure. In fact, the implant often ends up carrying most of the applied load and this leads to a low stress condition on bone resulting in interface failure [53]. Interface failure also happens as a result of excessive micromotion and stress at the bone-implant interface which prevents implant fixation resulting in failure. Since interface failure in implant design is a very important consideration, in Chapter 4, the minimization of this phenomenon will be considered as one of the objective functions in the optimization process. 


\subsubsection{Bone Loss}

Another common cause of dental implant failure is the bone loss happening at the bone surrounding the implant and can be observed around various designs of dental implants. Not only does it cause peri-implantitis and gingivitis, it also causes other problems like pain and loosening which may lead to the rejection of the implant [54]. Bone loss or bone resorption often occur because of mechanical bio-incompatibility of the implant with the surrounding bone and they can cause a gradual decrease of bone mass, strength and stiffness. One of the factors behind bone loss is the stress concentration around the implant. Bone needs to remain within a certain range of stress and strain for self-maintenance. While an implant replaces the tooth root, the implant carries the majority of load, causing a reduction of stress in surrounding bone which affects bone functionality. Therefore, to adapt to new conditions, bone starts resorbing, which leads to its low density and weakness. This is the second objective function considered in the design optimization problem discussed in this thesis. 


\section{Lattice Materials}

Lattice materials, their classification and some fundamental concepts in lattice material design will be discussed in this chapter. In addition, a brief review of the mechanical properties of lattice materials with focus on Octet-truss lattice type.

\subsection{Introduction}

A lattice material is a cellular solid that is built by tessellating a unit cell, composed of a small number of elements, in an infinite periodicity. Lattice materials are designed to expand the properties of the solid material which they are made from. These properties depend on the lattice cell topology and the material relative density $\bar{\rho}$ (the density of the lattice material divided by the density of the solid material which the lattice is built of)[55]. They are lightweight structures that have been popularly used in many different engineering applications. They are ubiquitous in biological systems, structural engineering and materials science. Lattice materials have excellent stiffness and strength-to-weight ratios. Current additive manufacturing technologies can build lattice structures with length scales ranging from nanometers to millimeters [37].

\subsection{Unit Cell and Cell Envelope}

The simplest repeating unit in a lattice structure is called a unit cell. A cell envelope is a virtual entity in a periodic lattice structure that represents the structural periodicity [55]. Two examples of planar lattice materials along with their unit cell finite structures and cell envelopes are shown in Figure 2.1. 


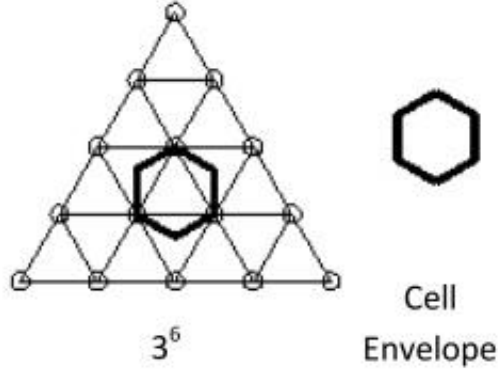

(a)

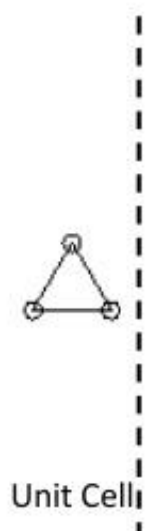

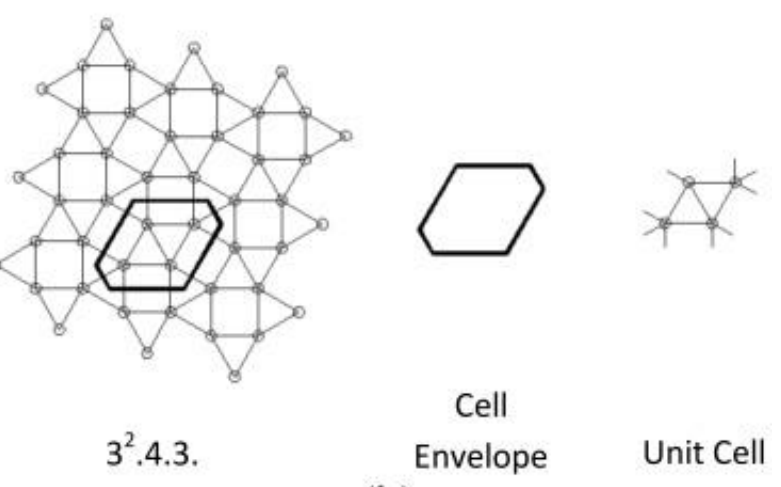

(b)

Figure 2.1: Microstructure of cellular materials: (a) Triangular 2D lattice structure; (b) 2D lattice structure with topology of Schlafli symbol [55]

\subsection{Classification of Lattice Structures}

Lattice structures can be categorized as planar structures (2D lattices) and spatial structures (3D lattices) [44]. For a 2D lattice material, the unit cell is repeated in a 2D plane and is infinitely extruded in the perpendicular plane. For a 3D lattice material, the unit cell is tessellated in three directions [45].

\subsubsection{Planar Lattice Structures}

A planar lattice is a $2 \mathrm{D}$ structure which can be made of one specific polygon type. In this case, it is called regular 2D lattice. Planar lattice materials can be used in electronic, photonic, optic, thermal and mechanical devices. Having similar properties along different crystal orientations are favorable for many conventional electronic and photonic applications. For example, transistors built along different crystal directions on the wafer will have the same characteristics, which is beneficial for controlling the variability and 
uniformity of the device performance across a large wafer [56]. Figure 2.2 shows three different 2D lattice types made of square, hexagonal and triangular unit cells.

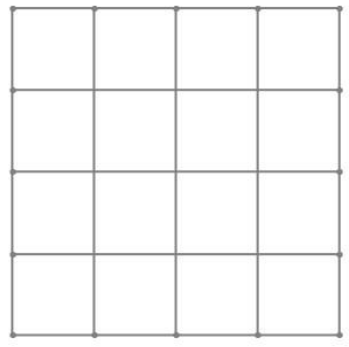

(a)

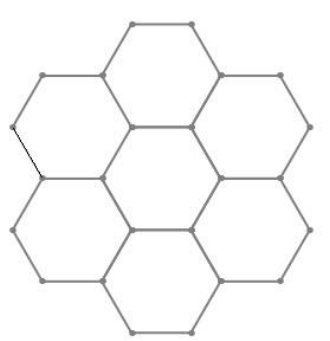

(b)

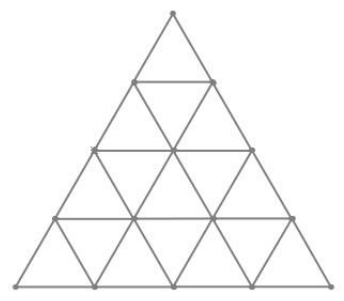

(c)

Figure 2.2: 2D regular planar lattices: (a) Square lattice; (b) Hexagonal lattice; (c)

Triangular lattice

In the semi-regular lattices, more than one regular polygon is used to tessellate the plane. An example of semi-regular lattice is the kagome lattice shown in Figure 2.3. The unit cell and cell envelop of this type of lattice are also presented in this figure.

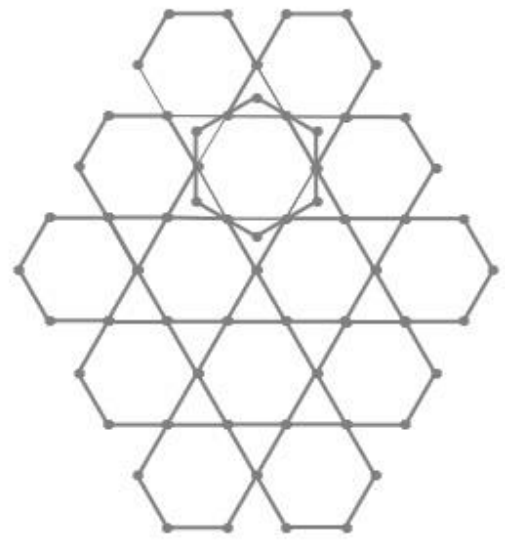

(a)

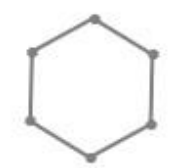

Cell envelop

(b)

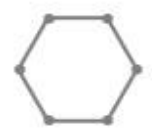

Unit Cell

(c)

Figure 2.3: (a) Kagome 2D lattice structure; (b) Cell envelop; (c) Unit cell 


\subsubsection{Spatial Lattice Structures}

Spatial lattice structures or 3D lattices can be built by tessellating polyhedra to fill the space. There are nine basic fundamental polyhedra as shown in Figure 2.4 that can be used to create a 3D lattice. For example, octet-truss lattice is made of a combination of tetrahedra and octahedra [55].

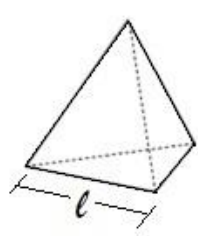

(a)

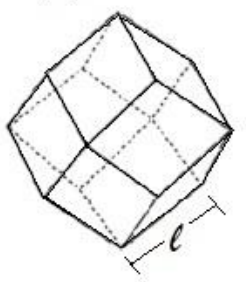

(f)

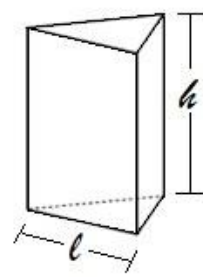

(b)

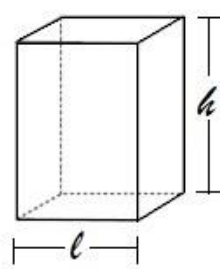

(c)

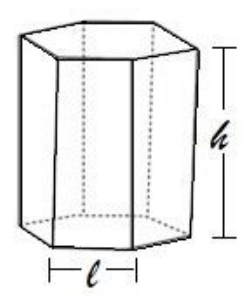

(d)

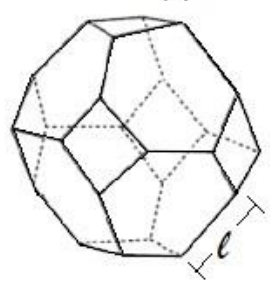

(h)

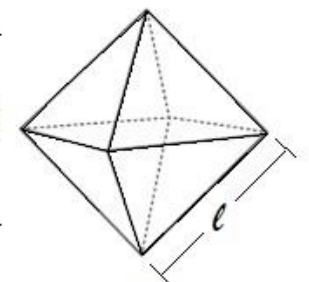

(e)

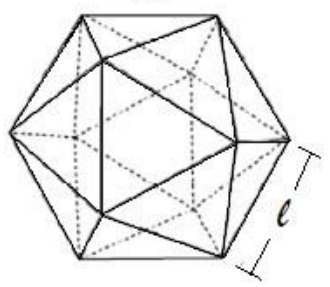

(i)

Figure 2.4: 3D polyhedral cells: (a) Tetrahedron; (b) Triangular prism; (c) Square prism; (d) Hegxagonal prism; (e) Octahedron; (f) Rhombic dodecahedron; (g) Dodecaherdon;

(h) Truncated octahedron; and (i) Iscosahedron [27]

\subsection{Mechanical Properties of Lattice Materials}

The mechanical properties of a lattice material strongly depend on its topology. Based on the topology, the deformation in a lattice material can be classified as stretching dominated or bending dominated. Cell walls deform predominantly by tension or compression for the former and by bending for the latter. The dominated mode of deformation affects the 
effective material properties of a lattice material [57]. In stretching dominated lattice materials (SDLM), the stiffness and strength scale in linear proportion to relative density and for bending dominated lattice materials (BDLM), they scale non-linearly as it shown in Table 2.1 [27].

Table 2.1: Stiffness and strength relation with relative density for lattice materials

\begin{tabular}{lll}
\hline Lattice type & Stiffness & Strength \\
\hline SDLM & $\propto \bar{\rho}$ & $\propto \bar{\rho}$ \\
\hline BDLM & $\propto \bar{\rho}^{2}$ & $\propto \bar{\rho}^{1.5}$
\end{tabular}

Because the relative density is less than unity, the stretching dominated lattice offers higher stiffness and strength per unit weight compared to the bending dominate one. For instance, for a $\bar{\rho}=0.1$, a stretching dominated topology would be 10 times stiffer and 3.16 times stronger than a bending dominated one [57]. The cell elements of a SDLM are essentially loaded in axial tension or compression. An idealized representation of the SDLM is an octet-truss structure with pin-joints that allow rotations. Figure 2.5 displays the structure of an octet-truss lattice unit cell.

\subsection{Octet-truss Lattice}

An octet-truss cell topology can be viewed as a regular octahedron core that is surrounded by eight regular tetrahedrons distributed on its eight faces. The cell has a Face Centered Cube (FCC) lattice structure with cubic symmetry generating a material with an isotropic behavior [58]. Figure 2.5 shows the microscopic topology of this lattice type. 


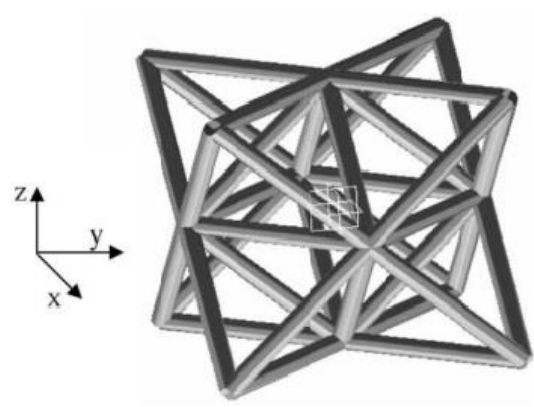

Figure 2.5: Structure of a regular octet-truss lattice unit cell [58]

\subsubsection{Elastic Properties of Octet-truss Lattice}

In this section, the effective mechanical properties of the octet-truss lattice material are presented. It is assumed that the cell element geometry of the lattice material is uniform with circular solid cross-section. The relative density of the regular octet-truss material $\left(\bar{\rho}_{L a}\right)$ can be expressed as [35]:

$$
\bar{\rho}_{L a}=\frac{\rho_{L a}}{\rho_{0}}=(6 \pi \sqrt{2})\left(\frac{a_{e}}{l_{e}}\right)^{2}
$$

and the relative Young's modulus of the octet-truss lattice material can be written as [35]:

$$
\bar{E}_{L a}=\frac{E_{L a}}{E_{0}}=\frac{2 \pi \sqrt{2}}{3}\left(\frac{a_{e}}{l_{e}}\right)^{2}
$$

where $a_{e}$ is the radius of the cell element cross section, $l_{e}$ is the element length, $\rho_{L a}$ is lattice material density, $\rho_{0}$ is the density of the solid material used to create the lattice material, $E_{L a}, \bar{E}_{L a}$ and $E_{0}$ are the Young's modulus of the lattice material, the relative Young's modulus of the lattice material and the Young's modulus of the solid material respectively [58].

By combining Equations (2.1) and (2.2), the relative Young's modulus of the lattice can be expressed as: 


$$
\bar{E}_{L a}=\frac{E_{L a}}{E_{0}}=\frac{1}{9} \bar{\rho}_{L a}
$$

The regular octet-truss cell geometry has a cubic symmetry, which generates a material with isotropic properties [59]. The Poisson's ratio of this lattice is found to be $v_{L a}=\frac{1}{3}$ [35]. The relative shear modulus of octet-truss lattice can be expressed as [35]:

$$
\bar{G}_{L a}=\frac{G_{L a}}{G_{0}}=\left[\frac{E_{L a}}{2\left(1+v_{L a}\right)}\right] / G_{0}=\frac{\bar{\rho}_{L a}}{9}
$$

where, $G_{0}$ is the shear modulus of the solid material used to build the lattice, $G_{L a}$ and $\bar{G}_{L a}$ are the shear modulus and the relative shear modulus of lattice material, respectively. The above equations will be used in Chapter 5 to design the interface zone of the implant with bone.

\subsubsection{Strength Properties of Octet-truss Lattice: Plastic Yield Strength}

The axial and shear yield strengths of the regular octet-truss lattice material can be formulated as [58]:

$$
\begin{gathered}
\bar{\sigma}_{y L a}=\frac{\sigma_{y L a}}{\sigma_{y 0}}=\pi \sqrt{2}\left(\frac{a_{e}}{l_{e}}\right)^{2} \\
\bar{\tau}_{y L a}=\frac{\tau_{y L a}}{\tau_{y 0}}=\pi \sqrt{2}\left(\frac{a_{e}}{l_{e}}\right)^{2}
\end{gathered}
$$

where $\sigma_{y 0}$ and $\sigma_{y L a}$ are the yield strengths of the solid material and the lattice material, respectively. Similarly, $\tau_{y 0}$ and $\tau_{y L a}$ are the shear strengths of. the solid material and the lattice material, respectively. 
By substituting Equation (2.1) into (2.5) and (2.6), the relative yield strengths of the lattice material can be expressed as:

$$
\begin{aligned}
& \bar{\sigma}_{y L a}=\frac{1}{6} \bar{\rho}_{L a} \\
& \bar{\tau}_{y L a}=\frac{1}{6} \bar{\rho}_{L a}
\end{aligned}
$$

\subsubsection{Strength Properties of Octet-truss Lattice: Elastic Buckling}

The critical buckling stress of the regular octet-truss lattice material can be expressed as [55]:

$$
\bar{\sigma}_{L a}^{c r}=\frac{\sigma_{L a}^{c r}}{\sigma_{y 0}}=\left(\frac{2 \pi^{3} \sqrt{2}}{3}\right)\left(\frac{E}{\sigma_{y 0}}\right)\left(\frac{a_{e}}{l_{e}}\right)^{4}
$$

where $\bar{\sigma}_{L a}$ and $\sigma_{L a}$ are the relative critical buckling strength and the critical buckling strength of the regular octet-truss lattice material, respectively. By combining Equations (2.1) and (2.7), the relative critical buckling can be obtained as:

$$
\bar{\sigma}_{L a}{ }^{c r}=\frac{\sigma_{L a}^{c r}}{\sigma_{y 0}}=\left(\frac{\pi}{54 \sqrt{2}}\right)\left(\frac{E}{\sigma_{y 0}}\right) \bar{\rho}_{L a}{ }^{2}
$$

To avoid failure by buckling, the following condition should be met [55]:

$$
\bar{\sigma}_{L a}^{c r} \geq \bar{\sigma}_{y L a}
$$

By substituting Equations (2.5) and (2.10) into (2.11), one gets:

$$
\bar{\rho}_{L a} \geq\left(\frac{6 \sqrt{2}}{\pi}\right)\left(\frac{\sigma_{y 0}}{E}\right)
$$


The equality form of above expression is the critical relative density and it can be determined by knowing the mechanical properties of the solid material which is going to be used for building the lattice. 


\section{Optimization}

Optimization is the process or methodology of making a design as functional and effective as possible. There are many different types of optimization methods used by engineers and scientists. In this chapter, topology optimization has been described as a useful tool for structural optimization and some examples are provided to better explain the concept of this method.

\subsection{Topology Optimization}

Topology optimization (TO) is a mathematical approach that aims to find the best material layout for a given set of loads and boundary conditions within a specified design space in order to optimize a given objective function subject to some constraints. Topology optimization is a very powerful tool in many different areas of engineering design, that can find the best possible material distribution within a given design space for a given set of loads, boundary conditions and constraints with respect to required objective functions. It is a well-established method to optimize the design of parts to create lightweight and low

cost structures. Application of this method to structural design, involves consideration of parameters such as stiffness, weight, displacements, buckling loads, frequencies, etc. Some of these can be considered as objective functions and others can be used to constrain the system performance. Minimization of mass, volume or compliance of a given system are some of the most common objective functions. Topology optimization provides the optimized distribution of the material throughout the design space which leads to significant weight reduction and improved structural performance of the system. 


\subsubsection{Introduction}

In structural design, topology optimization can be considered as an extension of shape and size optimization. In a shape optimization problem, the design domain is considered as the design variable and the goal is to find the optimum shape of this domain. In size optimization, the target is to find the optimal thickness distribution of a member which has been selected as the design variable. Topology optimization extends these two methods as it seeks to find the optimal domain of the design area subject to the functional requirements $[60]$.

Topology optimization is a powerful tool in many areas of design such as optics, electronics and structural mechanics. It is the most general type of structural optimization, being performed in the initial phases of the design. The whole feasible domain is considered and the aim is to find the most advantageous material distribution inside this domain, with respect to the design objectives [61]. This optimization method can be divided into two main subfields: Layout Optimization (LO) deals with grid-like structures having very low volume fractions whereas Generalized Shape Optimization (GSO) is concerned with higher volume fractions. In both problem classes, optimizing the solutions can be either exact/analytical or discretized/FE-based [62]. For very low volume fractions, Michell [63] derived formulae for structures with minimum weight given stress constraints on various design domains in the context of trusses. The basic principles of optimal layout theory were formulated and generalized by Prager and Rozvany [64]. Topology optimization for higher volume fractions was presented as GSO by Rozvany and Zhou [65]. In 1988, Bendsoe and Kikuchi $[51,52]$ introduced a homogenization method based on square and rectangular holes. The method of homogenization was used to generate optimal topologies in structural 
design. It was then developed into the Solid Isotropic Microstructure with Penalization (SIMP) method [68]. In this approach, the design domain is represented by values of materials in an element of a finite-element mesh and then the optimization is performed by assigning each element of the FEM of the design space a density, $\rho_{i}$, to be used as the design variable. The relative element density represents the amount of material present in the element. For example, a relative density of 1 corresponds to solid material and a relative density of 0 corresponds to a void.

Topology optimization is used in the initial phase of the design to obtain the optimum material distribution inside the available volume of a structure. It is important to know that the results obtained by topology optimization cannot be used directly, but need to be interpreted, because they just present how the material should be distributed over the design space [61].

A typical layout topology optimization problem, can be seen in Figure 3.1 which illustrates a possible result of a topology optimization problem for a cantilever beam subjected to a concentrated end load.
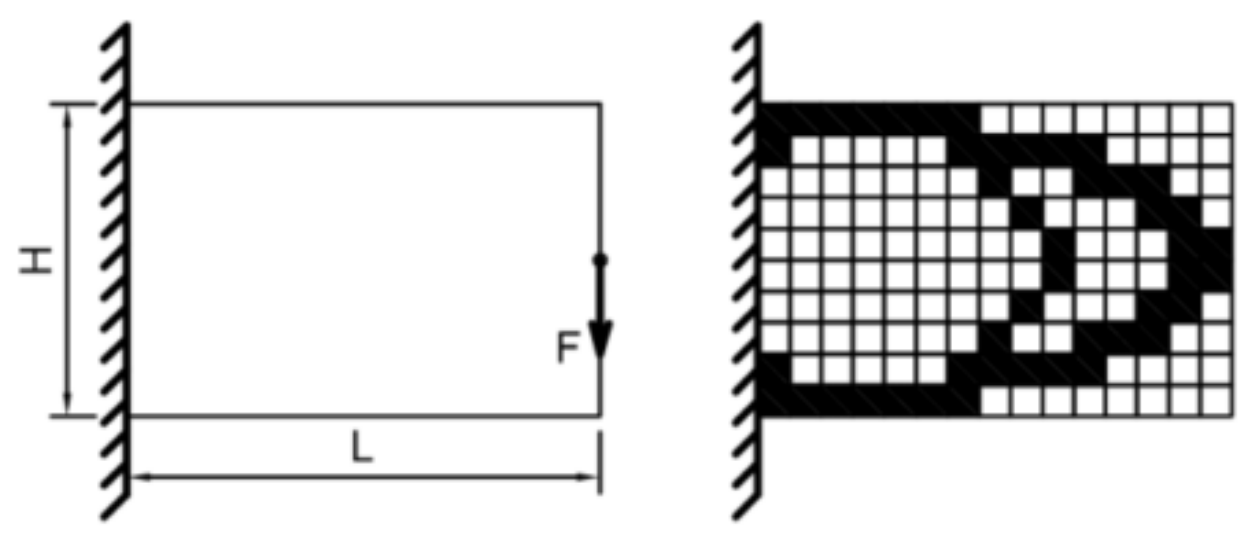

Figure 3.1: Typical topology optimization problem [61] 
The figure on the left represents the problem formulation and the feasible domain, while the one on the right shows the finite element grid, with each element being either black (corresponding to a zone where material is needed) or white (corresponding to a zone where material is not necessary) [61].

\subsubsection{Problem Statement of Topology Optimization}

In topological optimization, the ultimate goal is to find the material distribution that minimizes an objective function. One of the most common objective functions in structural optimization is the compliance of a system. Minimizing the compliance is equivalent to maximizing the global structural stiffness. Therefore, the general statement for a structural topology optimization problem can be expressed as:

$$
\begin{aligned}
& \text { Minimize } f^{T} u(x), \\
& \text { Subject to : } \sum_{i} x_{i}-V \leq 0, \\
& K(x) u(x)=f, \text { and } x_{i} \in[0,1]
\end{aligned}
$$

where $K(x)$ is the stiffness matrix as expressed in Equation (3.2), $u(x)$ is the displacement shown in Equation (3.2) and $t$ is the applied load.

$$
K(x)=\left[\begin{array}{cccc}
k_{11} & k_{12} & \ldots & k_{1 n} \\
k_{21} & k_{k 22} & \ldots & k_{2 n} \\
\ldots & \ldots & . . & \ldots \\
k_{n 1} & k_{n 2} & \ldots & k_{n n}
\end{array}\right]
$$




$$
u(x)=\left\{\begin{array}{l}
u_{1} \\
u_{2} \\
\ldots \\
u_{n}
\end{array}\right\}
$$

$V$ is volume here and $\sum_{i} x_{i}-V \leq 0$ presents the constraint for volume of the design space and it means that the total volume of the optimization model should not be higher than a specified value.

In this problem, $K(x) u(x)=f$ representing the finite-element formulation of the equations of linear elasticity and $x_{i}$ is the design variable. The objective function is used to minimize the compliance subject to an upper bound on the volume of the structure [69].

\subsection{Topology Optimization of Solid Beams}

Compared to size and shape optimization, topology optimization allows more freedom as no initial structure is required. Only the design space, the loads and the boundary conditions are required to be defined in order to find an optimized structure which satisfies the given constraints. In topology optimization, a fixed finite element mesh is used and one design variable is attributed to each element. The design variable determines if the corresponding element will represent structural material or a void [70].

In this optimization method, the finite element method is used to discretize the design domain and the finite element mesh represents the design variable.

In the following section two topology optimization examples of simple beams with different boundary conditions are depicted. 


\subsubsection{Rectangular Cantilever Solid Beam Topology Optimization}

In this section, a cantilever beam with the following dimension has been considered to implement the topology optimization by Altair OptiStruct software [71].

The beam is solid and made out of steel with $200 \mathrm{~mm}$ length $(L=200 \mathrm{~mm}), 100 \mathrm{~mm}$ crosssectional depth $(W=100 \mathrm{~mm})$ and $5 \mathrm{~mm}$ thickness $(t=5 \mathrm{~mm})$ as shown in Figure 3.2 .

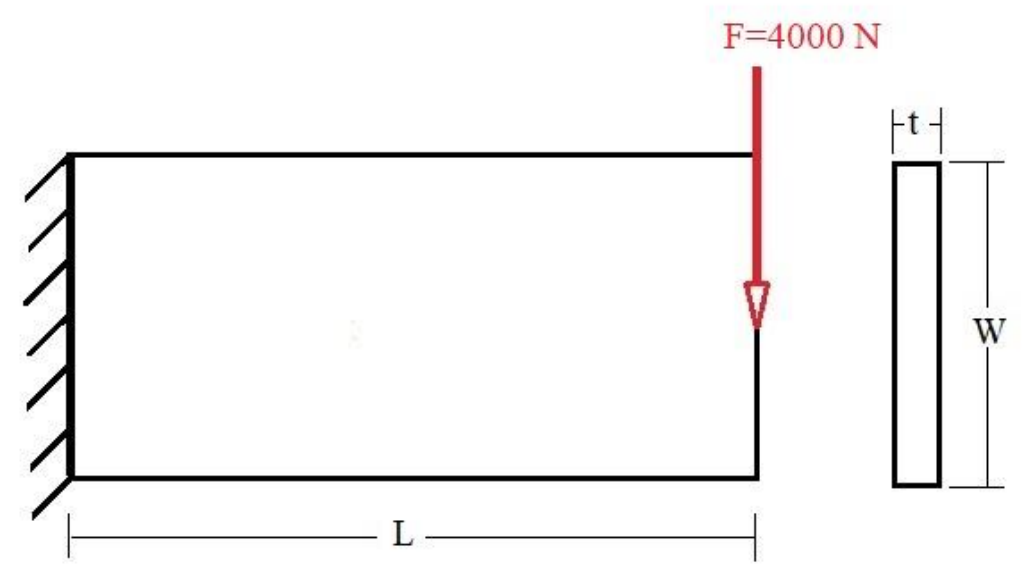

Figure 3.2: Rectangular cantilever beam subjected to a transverse load

The objective function of the topology optimization is to minimize the bending compliance of the structure subject to an upper bound of $30 \%$ for material volume fraction. The topology optimization result can be seen in Figure 3.3.

The topology optimization provides the optimal material layout by determining the best distribution of material according to the known load and boundary conditions in the design domain. As can be seen in Figure 3.3, the dark blue areas show where the material needs to be removed and the other colors show the density distribution over the design area. The results suggest that the optimal design can be a truss shape model instead of a complete solid beam. 


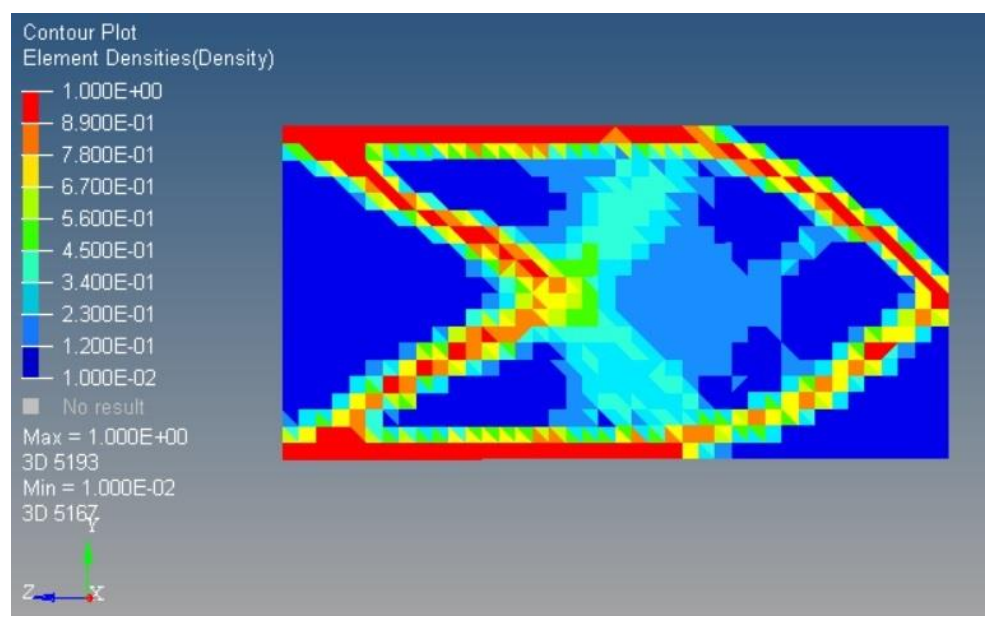

Figure 3.3: Topology optimization of a cantilever beam

\subsubsection{Rectangular Simply Supported Beam Topology Optimization}

The same beam as in previous section was considered to implement topology optimization for simply supported boundary conditions as can be seen in Figure 3.4. The dimensions of the beam are: $(L=200 \mathrm{~mm}, W=100 \mathrm{~mm}$ and $t=5 \mathrm{~mm})$.

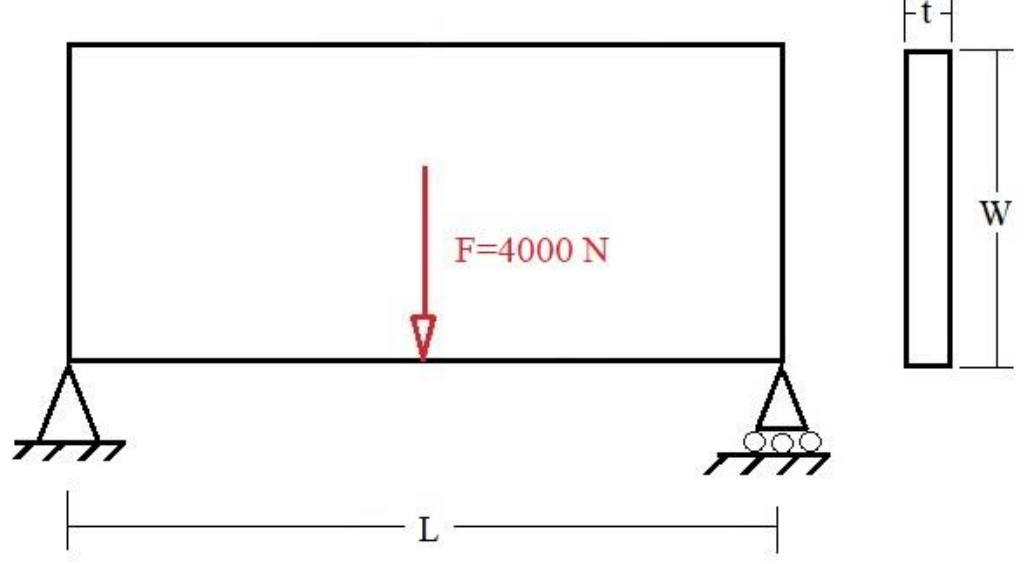

Figure 3.4: Simply supported beam subjected to transverse load 
The final result is shown in Figure 3.5. In this figure, the material layout of a simply supported beam has been determined and again the dark blue areas show where no material is needed and the other colors show the density distribution over the design area. For instance, the red color shows where there should be solid material. The optimal design would be a truss shape structure supported at both ends. Topology optimization, provides the best possible material distribution over the design area with the least amount of material that can be used to create a strong and stiff structure.

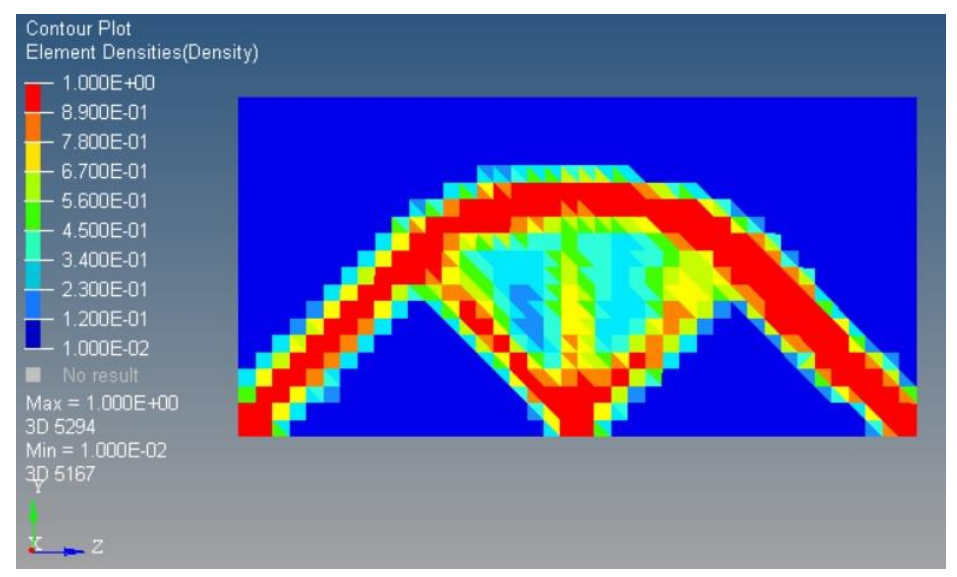

Figure 3.5: Topology optimization of a simply supported beam

\subsection{Multiscale Topology Optimization}

Multiscale topology optimization methods provide a material distribution solution to generate an optimal topology. In these methods, each finite element within the design domain should be defined as a design variable, allowing either a distributed variation in material density using solid isotropic microstructure with penalization (SIMP) [69], or a discrete void-solid (using bi-directional evolutionary structural optimization (BESO)) [72] material distribution. The SIMP algorithm for topology optimization penalizes intermediate densities to encourage discrete void-solid designs. However, this artificial 
penalization means that the optimized topology is less optimal than if the intermediate densities had not been penalized. If there were a way to manufacture these intermediate regions without a direct correlation to cost, then there would be no need to penalize them. It has been shown that microstructures like lattices or composites can be used to provide similar mechanical performance to these intermediate density regions [72]. As discussed earlier, mechanical properties of a lattice material are mainly governed by the topology of the unit cell, whose characteristic length should be at least one order of magnitude below that of the component, should the periodic structure be considered as behaving as a material. Several theoretical approaches have been proposed in the literature to calculate the mechanical properties of a lattice $[59,60]$. Figure 3.6 shows a solution to a simple cantilever plate optimization problem using the SIMP method but without the penalization and as it can be seen, there are large regions of intermediate density [72].

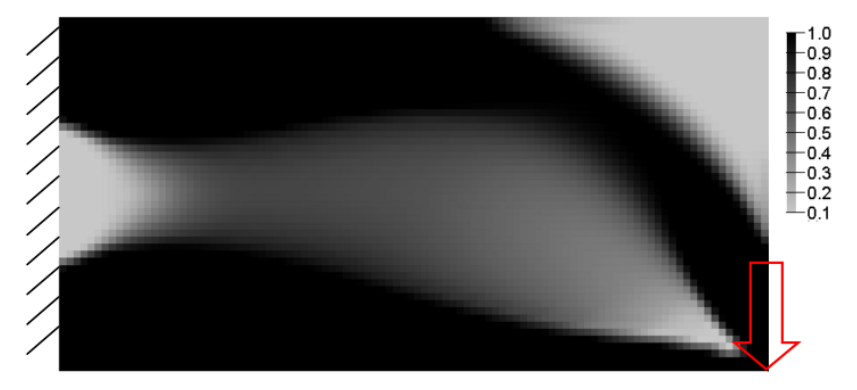

Figure 3.6: Optimized result for a cantilever plate problem using unpenalized SIMP [72]

\subsection{Lattice Topology Optimization}

Lattice optimization is a special form of multiscale topology optimization performed at a mesoscale. In this method, instead of discretizing all intermediate densities, as in topology optimization, any intermediate densities between predetermined limits can be transformed 
into a lattice unit cell. Once again, Altair OptiStruct software [71] was employed to perform lattice optimization for the same rectangular cantilever as well as simply supported beam presented in Sections 3.2.1 and 3.2.2. When lattice optimization is performed, any element in the design space, which is converted into lattice, is replaced with a lattice unit cell consisting of several 1-dimensional beam elements meeting at central nodes. The unit cells can be any arrangement of BCC (Body Centered Cubic), FCC (Force Centered Cube) cells, octet truss and others. based on the optimization setup at the beginning. Below, the lattice optimization of the simply supported and the cantilevered beam are presented.

\subsubsection{Rectangular Cantilever Beam Lattice Optimization}

The beam in Section 3.2.1 with the same objective function and constraint was optimized based on lattice optimization capability of OptiStruct [71]. Figure 3.7 shows the material layout for the new optimization. The contour plot on the left side of the image shows the element density distribution over the design space. The red color represents a density of 1 implying that in red areas the material is solid. Dark blue areas represent areas with almost zero element density, which means no material is needed and these areas can be replaced by voids. The other colors represent the lattice zone representing lattice unit cells with different densities. Each color in the design space has a specific density and it can be replaced by a lattice that has the same density. The final product of lattice optimization for a cantilever beam can be seen in Figure 3.7. As it is shown the red color area in design space has been replaced by solid unit cells, other areas with intermediate densities have been replaced by lattice unit cells with densities corresponding to each region and the dark blue areas converted to voids. 


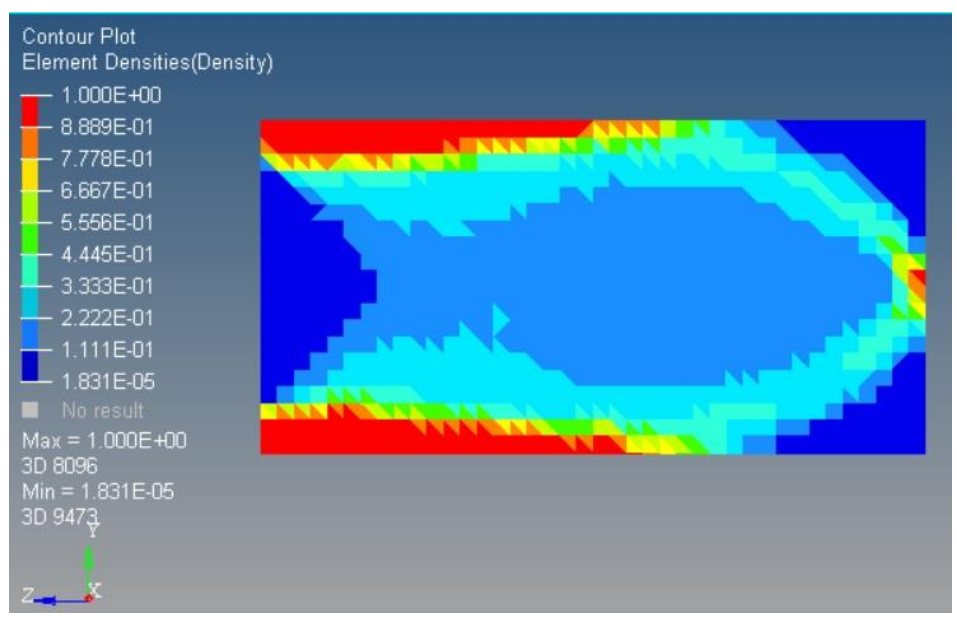

Figure 3.7: Lattice optimization of a cantilever beam (material layout)

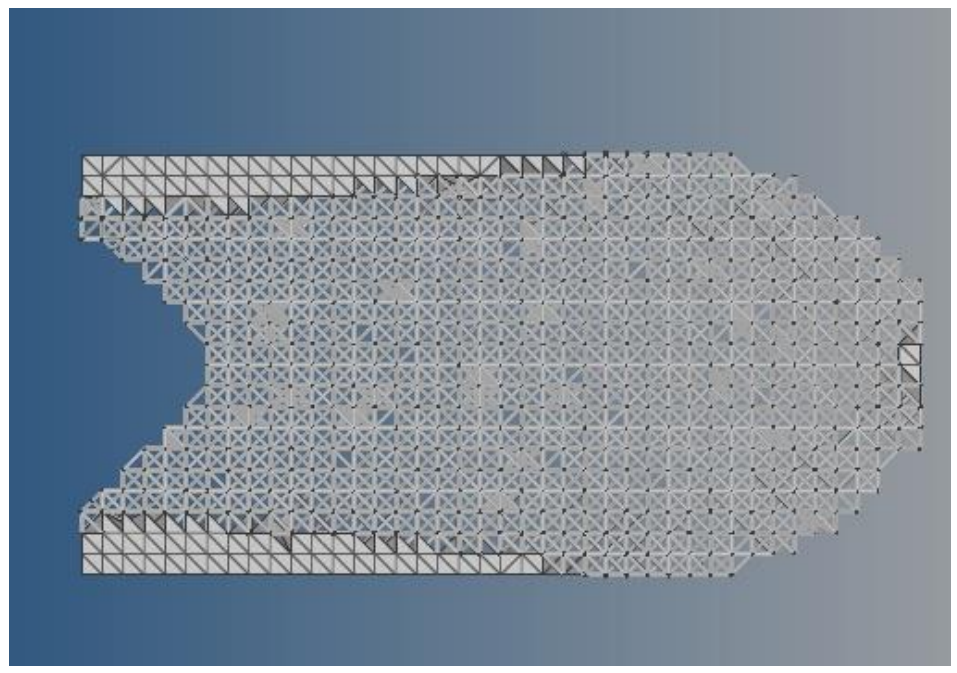

Figure 3.8: Lattice optimized cantilever beam

The final amount of mass that can be used in cantilevered beam design for both topology and multiscale optimization are depicted in Table 3.1. As expected, the weight saving in multiscale optimization is higher than topology optimization. This comes from the fact that multiscale optimization is capable of creating a structure with intermediate densities by 
employing lattice unit cells, while in topology optimization, the intermediate densities have to be penalized and converted to solid elements.

Table 3.1: Summary of mass reduction in topology and multiscale optimization of cantilevered beam

\begin{tabular}{lccc}
\hline & Solid model & Topology optimization & Multiscale lattice optimization \\
\hline Mass (gr) & 785 & 313.3 & 258.1 \\
\hline Weight saving & - & $60.1 \%$ & $67 \%$
\end{tabular}

\subsubsection{Rectangular Simply Supported Beam Lattice Optimization}

In this section the simply supported beam in Section 3.2.2 was optimized based on lattice optimization in Altair Optistruct [71]. The material distribution can be seen in Figure 3.9. Again different colors represent the intermediate densities. Dark blue is used in locations where no material is needed, and voids can be introduced. Other areas can be replaced by lattice unit cells having the same relative density. The final optimization product is shown in Figure 3.10.

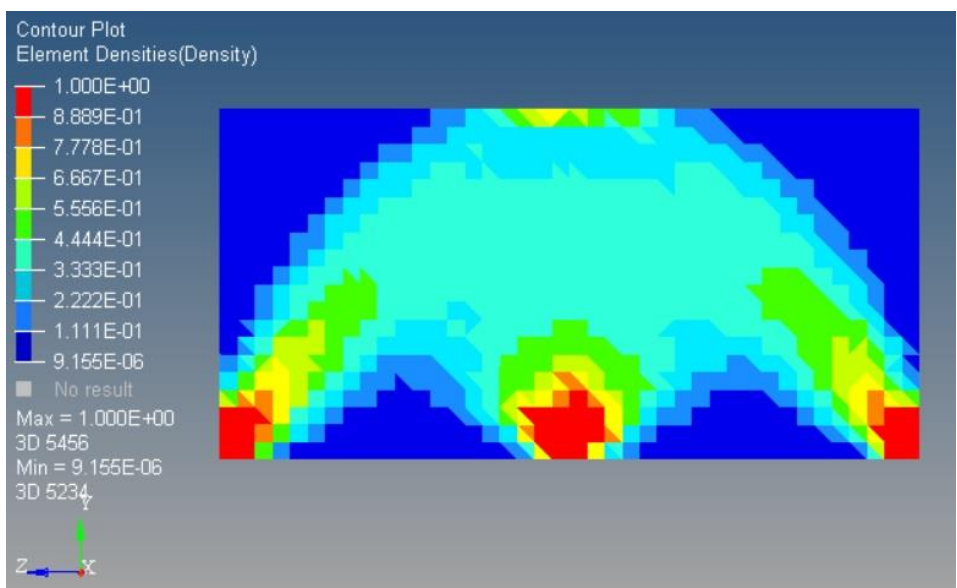

Figure 3.9: Lattice optimization of a simply supported beam (material layout) 


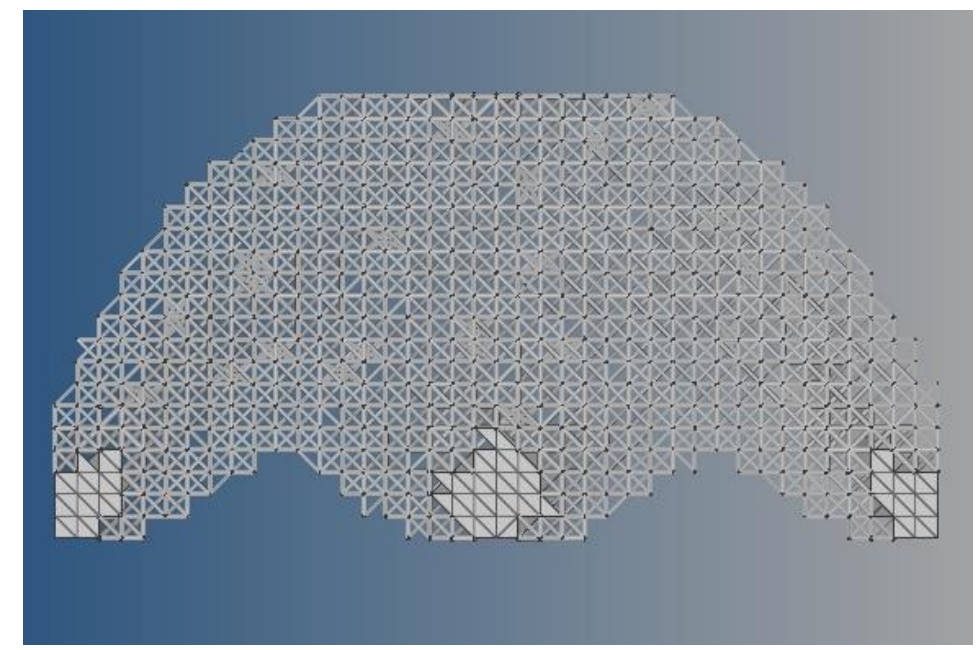

Figure 3.10: Lattice optimized simply supported beam

The mass calculation for both topology and multiscale optimization shows that the weight saving in multiscale optimization is higher than topology optimization. Table 3.2 summarizes the amount of mass than can be used to design the simply supported beam where considering these two methods, and again the results imply that, multiscale optimization provides a lighter structure than topology optimization. Therefore, it can be employed when weight reduction is critically significant.

Table 3.2: Summary of mass reduction in topology and multiscale optimization of simply supported beam

\begin{tabular}{lccc} 
& Solid model & Topology optimization & Multiscale lattice optimization \\
\hline Mass (gr) & 785 & 328.2 & 275.1 \\
\hline Weight saving & - & $58.2 \%$ & $65 \%$ \\
\hline
\end{tabular}




\subsection{Lattice Topology Optimization of Dental Implant with Minimal Compliance}

In this section, the design optimization of a dental implant based on minimum compliance as the objective function is presented. A 3D CAD model of a mandibular section of bone with $21 \mathrm{~mm}$ height and $20 \mathrm{~mm}$ width, representing the section of the mandible in the second premolar region, was created [75], as shown in Figure 3.11. The dental implant dimensions are also depicted in Figure 3.12.
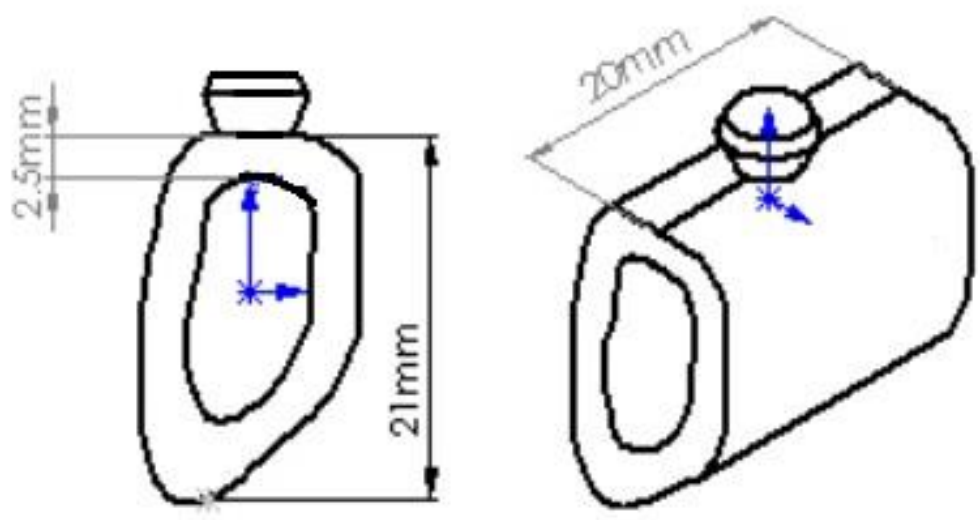

Figure 3.11: Geometry and dimension of bone segment

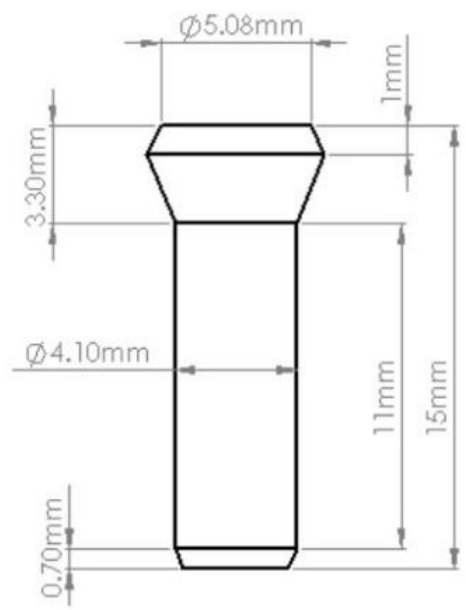

Figure 3.12: Geometry and dimension of implant 
As shown in Figure 3.13, the model consisted of a spongy core, representing cancellous bone, surrounded by an average thickness of $2.5 \mathrm{~mm}$ cortical bone. The implant was considered as a single-piece part of $15 \mathrm{~mm}$ length and $4.1 \mathrm{~mm}$ diameter in the cylindrical part. The bone was fixed at both ends and the implant loaded with forces of $17.1 \mathrm{~N}, 114.6$ $\mathrm{N}$ and $23.4 \mathrm{~N}$ in the lingual, axial, and mesiodistal directions [76,77], respectively as described in Figure 3.14. The force magnitudes were chosen based on the work of Mericske-Stern [76].

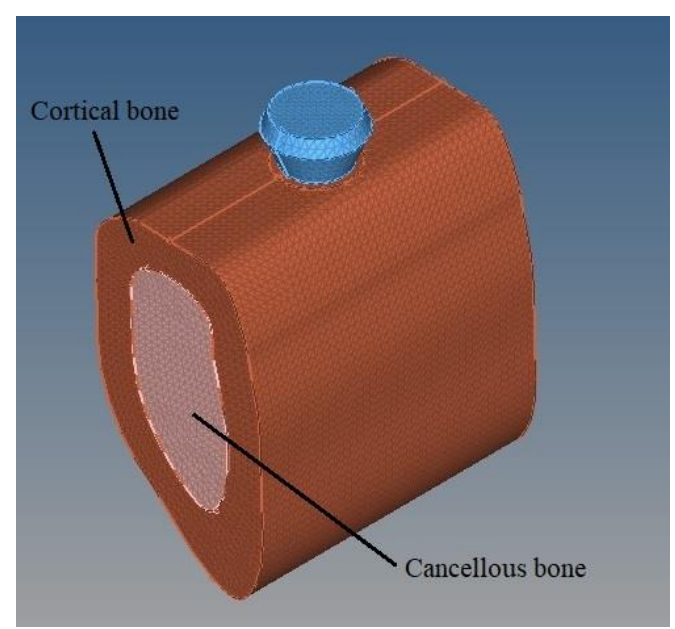

Figure 3.13: FEA model of bone and implant

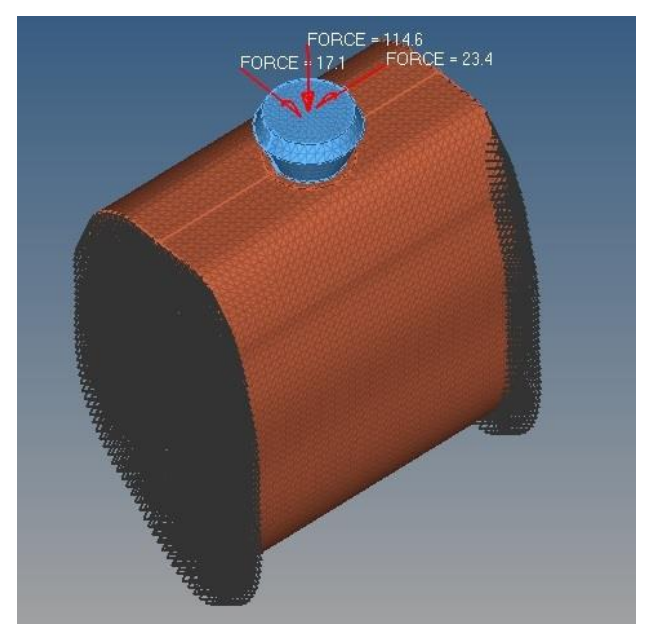

Figure 3.14: FEA model of the bone and implant and loading conditions

The objective function in this optimization is the compliance of the implant and the constraint is assumed that the volume fraction of the implant after optimization should not be higher than $50 \%$. The optimization results can be seen in Figure 3.15. The bone has been considered as a non-design space and the whole implant is the design space. 


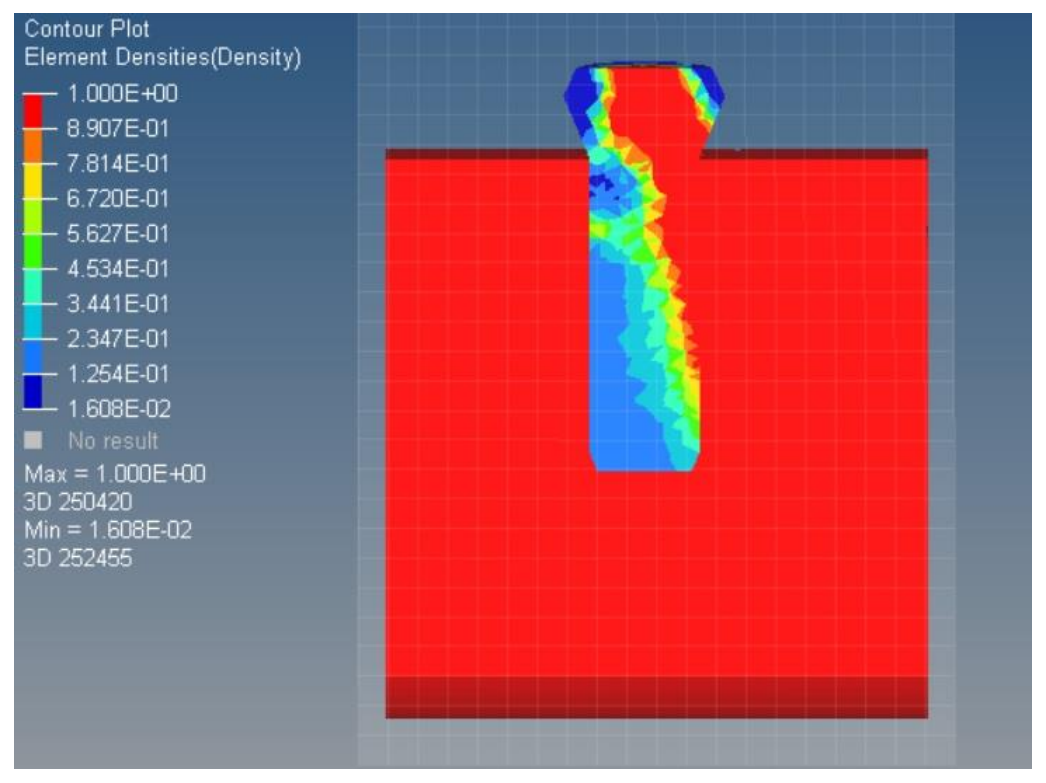

Figure 3.15: Material layout of dental implant after optimization

Different colors in the design space again represent the intermediate densities and they can be replaced by lattice unit cells with the corresponding relative densities. The final product of optimization is depicted in Figure 3.16. As can be seen, it is a multiscale optimized design made out of solid and lattice materials.

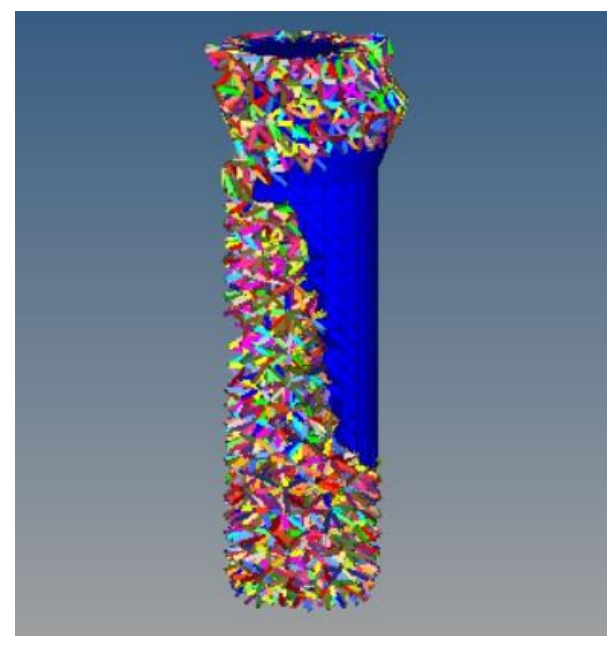

Figure 3.16: Final product of implant optimization 
In the next chapter, another method is presented to perform the optimization of the implant based on two objective functions including interface failure and the bone loss. 


\section{Multiscale and Multi-Objective Design Optimization of Dental Implant Employing Lattice Material}

This chapter presents a novel design for dental implant employing lattice materials. A multiscale and multi-objective design optimization framework based on the finite element method is presented to, primarily, minimize the bone/implant interface failure and bone loss and, secondarily, to minimize the implant weight which is important for the cost.

\subsection{Introduction}

As described, before a lattice material is a class of open cell engineered cellular solid that is periodically structured and optimized for different applications. In this chapter, we employ lattice material to design a new dental implant. A multiscale and multi-objective design optimization framework based on the finite element method was developed to minimize the bone/implant interface failure and bone loss while minimizing the implant weight. Here, we assumed the implant was made of a lattice part (interface zone with the bone) and a solid part (implant core). The design variables included the microscopic parameters of the lattice unit cell as well as thickness of the interface zone. Additively manufactured dense Titanium, a biocompatible material, is used for the new implant manufacturing, eliminating the need for a biocompatible coating.

\subsection{Computational Model Development}

In this section, a 3D CAD model of a segment of jaw bone and implant was developed, then the model was imported into Altair Optistruct Hyperworks finite element software [71] to create the finite element model. The FEM model consisted of total 26,575 nodes and 116,683 tetrahedron elements with $0.5 \mathrm{~mm}$ size; 35,269 elements for cancellous bone, 
74,469 elements for cortical bone and 6,945 elements for dental implant. Then boundary conditions were applied and the properties of the lattice zone were found according to Section 2.5 and used as the material properties in FEA. Then static analysis was performed and the results were used to calculate and evaluate the objective functions.

\subsubsection{Geometry and Finite Element Modeling}

A 3D model of a mandibular section of bone of $21 \mathrm{~mm}$ height and $20 \mathrm{~mm}$ width, as can be seen in Figure 3.11 representing the section of the mandible in the second premolar region shown in Figure 4.1, was modeled. The model consisted of a spongy core, called cancellous bone, surrounded by $2.5 \mathrm{~mm}$ thickness of cortical bone envelop as presented in Figure 4.2 . On the other hand, the implant was considered as a single-piece part of $15 \mathrm{~mm}$ length and $4.1 \mathrm{~mm}$ diameter in the cylindrical part according to Figure 3.12. The cylindrical section of the implant was assumed to be the part that contained the interface zone with the bone. This is the part of the implant that was considered for the lattice structure design.

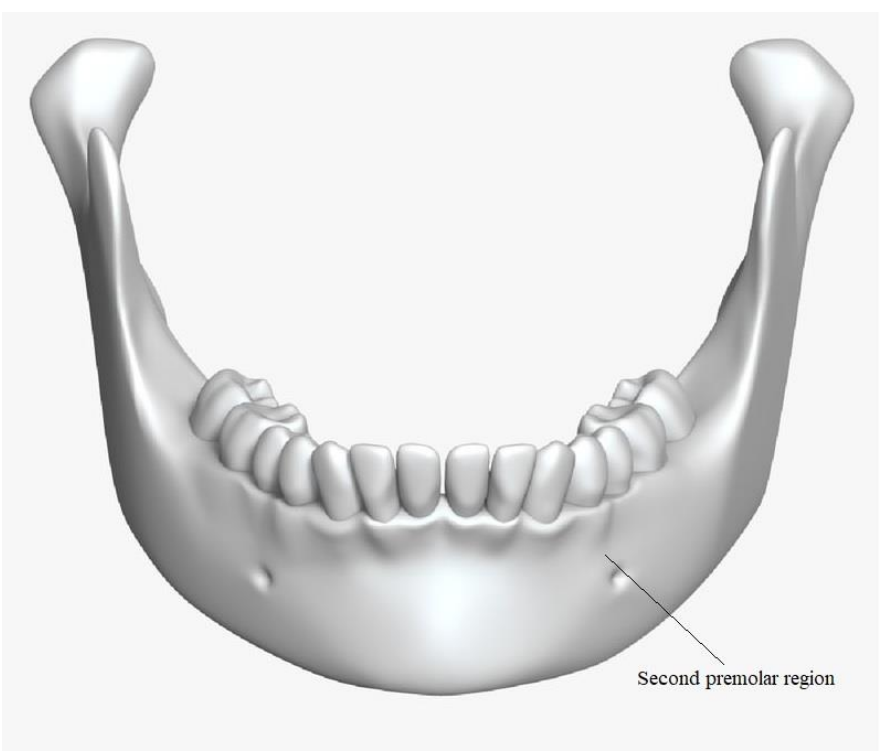

Figure 4.1: Lower mandible (second premolar region is shown in the picture) [78] 


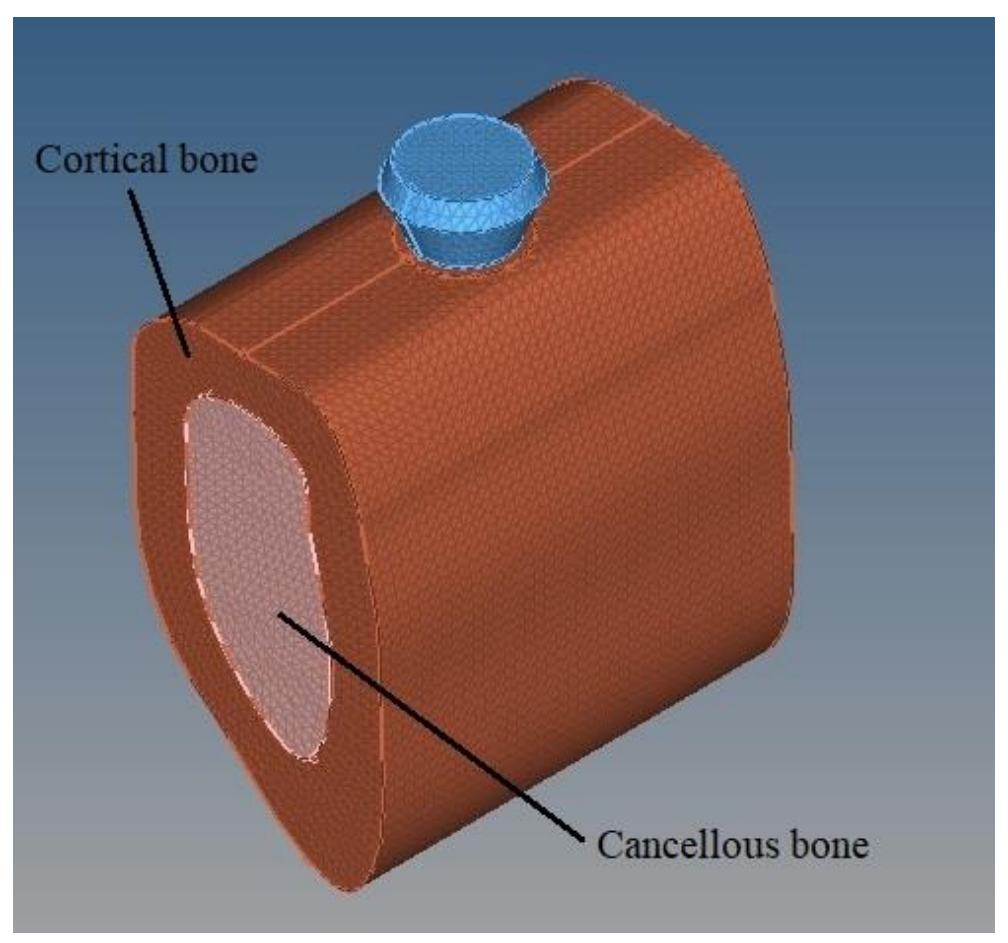

Figure 4.2: FEA model of bone and implant

The mechanical properties and densities of the materials modelled in this study are tabulated in Table 4.1.

Table 4.1: Mechanical properties of materials used in this study

\begin{tabular}{lcccc}
\hline Material & $\begin{array}{c}\text { Elastic Modulus } \\
(\mathrm{GPa})\end{array}$ & Poisson ratio, $v$ & $\begin{array}{c}\text { Density } \\
\left(\mathrm{gr} / \mathrm{cm}^{3}\right)\end{array}$ & $\begin{array}{c}\text { Yield stress } \\
(\mathrm{MPa})\end{array}$ \\
\hline Dense titanium [75] & 103.4 & 0.35 & 4.5 & 880 \\
Cortical bone [75] & 13.7 & 0.3 & 2.17 & 114 \\
Cancellous bone [75] & 1.37 & 0.3 & 1.0 & 5.4 \\
Tooth (dentin) [79] & 18.6 & 0.3 & 2.2 & 146 \\
\hline
\end{tabular}

The 3D CAD model, was imported into Altair Optistruct Hyperworks finite element software to perform the stress analysis. The stress analysis results then were used to 
calculate the objective functions. In Altair Optistruct, the cylindrical part of the implant was divided into two zones. The solid core was surrounded by the interface zone with the bone, made of lattice structure. The thickness of the interface zone as well as the lattice properties were the design variables of the optimization problem. Contact surfaces were defined between the bone and the implant to simulate the bonding between them while it was assumed that there was perfect bonding between the bone and the implant. The thickness of the interface zone varied between $0.2 \mathrm{~mm}$ to $2 \mathrm{~mm}$, since the implant diameter was $4.1 \mathrm{~mm}$. The interface zone thickness could be any value equal or less than implant radius. The minimum value of the thickness was selected to be greater than the minimum resolution $t_{\min }$ offered by a given manufacturing process. For example, $t_{\min }$ is $100 \mu \mathrm{m}$ and $70 \mu \mathrm{m}$, respectively, for SLM and SLA $[47,80]$. In order to have the bone ingrowth into the pores, the pore size also needs to be higher than $85 \mu \mathrm{m}$ [81]. The relative density of the lattice part was allowed to vary from 0.12 to 0.5 for various design. The lowest value of the relative density of the lattice material was selected to be 0.12 , so that the Young's modulus of the lattice material would be at least equal to or higher than the lowest Young's modulus of the bone.

\subsubsection{Boundary Conditions and Critical Loading}

The implant was loaded with forces of $17.1 \mathrm{~N}, 114.6 \mathrm{~N}$ and $23.4 \mathrm{~N}$ in the lingual, axial, and mesiodistal directions, respectively [77], as shown in Figure 4.3. These loads were suggested based on the work of Mericske-Stern and et al [76]. They measured the force transmission onto implants in vivo by means of piezoelectric transducers and then measured the maximum loads for different types of denture anchorage in the case of maximum biting during biting on a bite plate, grinding and chewing bread. These forces 
were used by many other researchers to do the static analysis of the implant. In this work, the same loads were applied to the implant. Finally, the bone was fixed at both ends and model prepared for analysis.

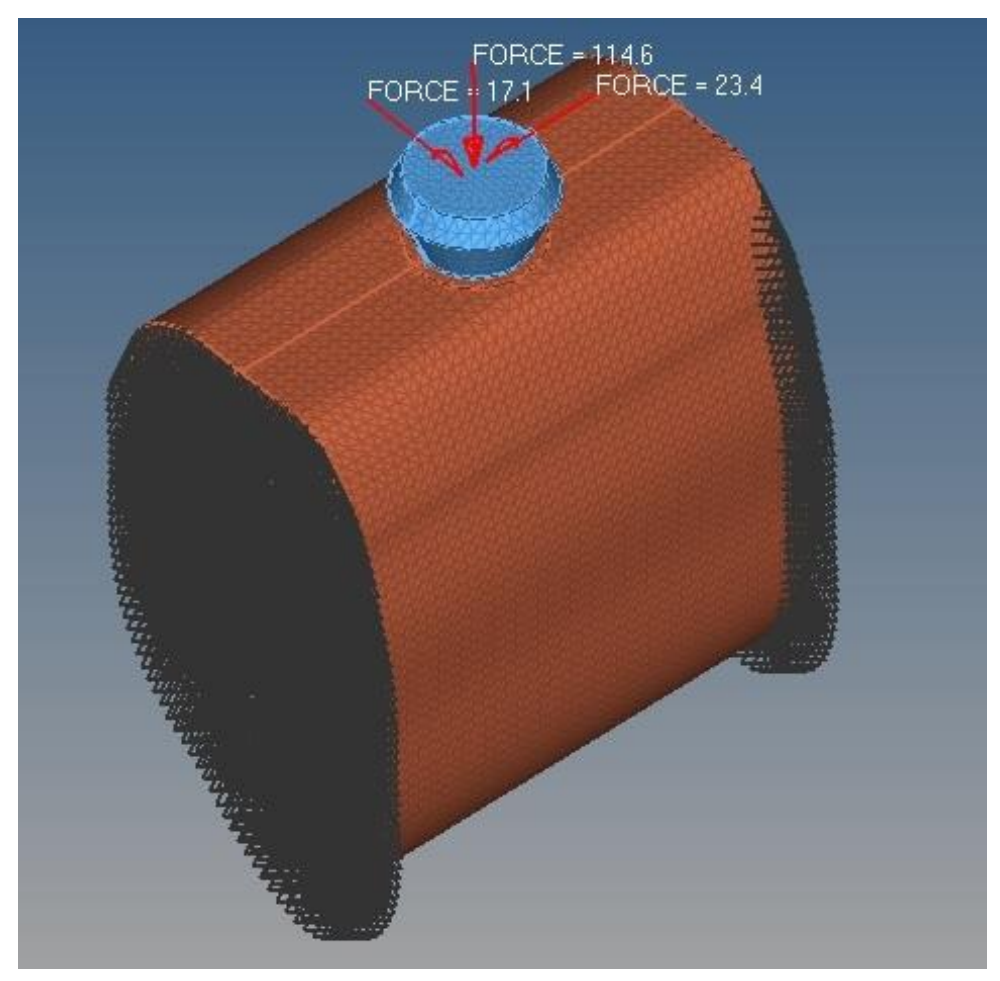

Figure 4.3: Applied loads and boundary conditions of FEM model.

\subsection{Effective Mechanical Properties of the Lattice Material}

Relative density of the lattice material as well as the thickness of the interface zone are considered as the design variables in this study and isotropic octet-truss lattice material was selected to tessellate the interface zone as shown in Figure 2.5.

As already discussed in Section 2.5, the relative density of the octet-truss lattice and its Young's modulus can be expressed as: 


$$
\begin{gathered}
\bar{\rho}_{L a}=\frac{\rho_{L a}}{\rho_{0}} \\
\bar{E}_{L a}=\frac{E_{L a}}{E_{0}}
\end{gathered}
$$

By combining these two, we can find the relation between Young's modulus and relative density of the lattice.

$$
\bar{E}_{L a}=\frac{E_{L}}{E_{0}}=\frac{1}{9} \bar{\rho}_{L a}
$$

The relative density of the lattice will be considered as one of the design variable to control the interface stiffness.

As mentioned before in Section 2.5.3, to avoid any elastic buckling the following inequality should also be satisfied:

$$
\bar{\rho}_{L a} \geq\left(\frac{6 \sqrt{2}}{\pi}\right)\left(\frac{\sigma_{y 0}}{E}\right)
$$

Where $\sigma_{y 0}$ is the yield strength of the solid material, which is Titanium here. According to Table 4.1:

$\sigma_{y 0}=880 \mathrm{MPa}$ and $E=103400 \mathrm{MPa}$. By substituting these values into Equation (4.4) one may obtain: $\bar{\rho}_{L a} \geq 0.023$.

To avoid any mechanical failure, the Von Mises stress of the lattice should be less than the yield strength of the lattice. As illustrated before in Section 2.5.2, the relative yield strength of octet-truss lattice can be expressed as:

$$
\bar{\sigma}_{y L a}=\frac{1}{6} \bar{\rho}_{L a}
$$

By using this equation, the yield strength for any relative density of the lattice can be determined. After performing the static analysis on implant and bone, the maximum Von 
Mises stress in the lattice zone should be compared with the yield strength of the lattice to make sure no failure occurs.

\subsection{Optimization of Implant with Minimum Interface Failure and Bone Loss}

The objective functions for optimization in this study are minimizing the bone loss and interface failure in order to reduce the chance of failure in implants and minimization of implant weight which is important for overall production cost. In this section, these functions are described.

\subsubsection{Bone Loss Objective Function}

As discussed in Chapter 2 bone loss happens because of the mechanical bio-incompatibility of the implant with the surrounding bone and it can cause a gradual decrease of bone mass, strength and stiffness.

Bone loss can be represented by the amount of bone that is going to be under-loaded in the presence of the implant.

To estimate the bone loss around the implant, the amount of bone that is underloaded needs to be assessed. Bone can be considered locally underloaded when its local strain energy $\left(U_{i}\right)$ per unit of bone mass $(\rho)$, averaged over $n$ loading cases is beneath the local reference value $S_{\text {ref }}$, which is the value of $S$ when there is no implant and is expressed as [53]:

$$
S=\frac{1}{n} \sum_{i=1}^{n} \frac{U_{i}}{\rho}
$$

However not all underloading leads to bone loss [53]. In fact, bone resorption happens

when the local value of $S$ is beneath the value of $(1-s) S_{\text {ref }}$ [43]. The value of $s$ representing the dead zone, was assumed to be 0.5 in this expression [53]. 
The resorbed bone mass fraction can be expressed as [53]:

$$
m_{r}(b)=\frac{1}{M} \int_{V} g(S(b)) \rho d V
$$

where $M$ and $V$ are the original bone mass and volume in bone interface and $g(S(b))$ is the resorptive function, so that [43]:

$$
g(S(b))=\left\{\begin{array}{lll}
1 & \text { when } & S<(1-s) S_{\text {ref }} \\
0 & \text { when } & S>(1-s) S_{\text {ref }}
\end{array}\right.
$$

To define the bone loss around the implant the number of elements that are unloaded can be counted and used to calculate the bone loss fraction. In this work, for simulation, the local strain energy of the interface elements, was determined by applying the properties of the tooth to the implant. Then the static analysis was performed and reference strain energy density of the bone interface elements, were extracted and used to evaluate the resorptive function in Equation (4.8) by comparing to the local strain energy density of interface elements in different models and finally bone loss was calculated by the total number of underloaded elements.

\subsubsection{Interface Failure Objective Function}

The other objective function was the interface failure which can be identified as the

probability of mechanical failure at the bone implant interface and can be expressed as [43]:

$$
F(b)=\int_{\Omega} f(\sigma) d \Omega
$$


where $F(b)$ is the global interface failure function index, $\sigma$ is the interface stress depending on the design variable. In Equation (4.9), $f(\sigma)$ is the interface failure probability and can be defined by the multi-axial Hoffman failure criterion [82]:

$$
f(\sigma)=\frac{1}{S_{t} S_{c}} \sigma_{n}^{2}+\left(\frac{1}{S_{t}}-\frac{1}{S_{c}}\right) \sigma_{n}+\frac{1}{S_{s}^{2}} \tau^{2}
$$

where $S_{t}$ and $S_{c}$ are the uniaxial tensile and compressive strengths, respectively, $S_{s}$ is the shear strength, and $\sigma_{n}$ and $\tau$ are normal and shear stresses at the bone-implant interface. If $f(\sigma)>>1$, a high probability of failure is expected, and if $f(\sigma)<<1$ the risk of interface failure is low. $S_{t}, S_{c}$ and $S_{s}$ each is expressed as a function of bone density as follows [83]:

$$
S_{t}=14.5 \rho^{1.71}, S_{c}=32.4 \rho^{1.85}, S_{s}=21.6 \rho^{1.65}
$$

\subsubsection{Optimization Problem}

Finally, the optimization problem can be formulated as:

Minimize: $\left\{\begin{array}{lr}m_{r}(b) & \text { Bone Loss } \\ F(b) & \text { Interface Failure } \\ m & \text { implant mass }\end{array}\right.$

Subject to $\left\{\begin{aligned} \bar{\rho}_{L a} & \geq 0.12(\text { Lattice relative density }) \\ 0.2 & <t<2(\text { Interface zone thickness }) \\ \sigma_{\text {VonMises }(L a t i c e)} & \leq \frac{1}{6} \bar{\rho}_{L a} \sigma_{\text {yield }(T i)} \text { To avoid material failure } \\ \bar{\rho}_{L a} & \geq \frac{6 \sqrt{2}}{\pi}\left(\frac{\left.\sigma_{\text {yield }(T i)}\right)}{E_{T i}}\right) \text { To avoid buckling }\end{aligned}\right.$ 
where $b$ is the design variables vector which includes the interface zone thickness and the relative density of lattice. $\sigma_{\text {yield }(T i)}$ and $E_{T i}$ are Titanium yield strength and Young's modulus, respectively. These values are given in Table 4.1.

\subsection{Results}

Finite element models for different thicknesses and different relative densities were developed and the FEA results for normal stress, shear stress and local strain energy densities were used to evaluate the stress in the implant and calculate the interface failure and bone loss for each model. The interface thicknesses between $0.2-2 \mathrm{~mm}$ were considered. The results showed that at thicknesses greater than $1.2 \mathrm{~mm}$, the bone loss and interface failure increased significantly.

Therefore, thicknesses between 0.2-1.2 $\mathrm{mm}$ were selected to study the bone loss and interface failure in order to determine the optimum thickness and relative density.

\subsubsection{Maximum Von Mises Stress in Lattice Zone}

To do the static analysis, different FEA models for different relative densities and different interface thicknesses were created. The static analysis was performed for each model. Then the Von Mises stress in lattice zone was compared to the yield strength of the lattice for each individual model to make sure that no failure happened in the lattice part. The results of maximum Von Mises stress for various combinations of relative density and lattice thicknesses are presented in Table 4.2 . 
Table 4.2: Maximum Von Mises stress $(\mathrm{MPa})$ in lattice zone for different relative densities and thicknesses

\begin{tabular}{lccccccc}
\hline \multirow{2}{*}{ Relative density } & \multicolumn{2}{l}{ Thickness of lattice $(\mathbf{m m})$} & \multicolumn{2}{c}{$\begin{array}{c}\text { Lattice yield strength } \\
\text { (MPa) }\end{array}$} \\
\cline { 2 - 7 } & 0.2 & 0.4 & 0.6 & 0.8 & 1.0 & 1.2 & 17.600 \\
0.12 & 2.2160 & 1.8500 & 1.7660 & 1.6770 & 2.1600 & 2.0110 & 22.000 \\
0.15 & 2.4770 & 2.2070 & 2.0760 & 2.0000 & 2.5740 & 2.3700 & 29.333 \\
0.20 & 2.8910 & 2.6980 & 2.5320 & 2.4910 & 3.1990 & 2.9150 & 36.667 \\
0.25 & 3.2300 & 3.0860 & 2.9300 & 2.9360 & 3.7610 & 3.3840 & 44.000 \\
0.30 & 3.5160 & 3.3950 & 3.2850 & 3.3440 & 4.2710 & 3.8000 & 51.333 \\
0.35 & 3.7640 & 3.6460 & 3.6070 & 3.7220 & 4.7380 & 4.1980 & 58.667 \\
0.40 & 3.9820 & 3.8530 & 3.9010 & 4.0750 & 5.1690 & 4.5700 & 66.000 \\
0.45 & 4.1790 & 4.0250 & 4.1730 & 4.4070 & 5.5700 & 4.9130 & 73.333 \\
0.50 & 4.3580 & 4.1690 & 4.4260 & 4.7190 & 5.9440 & 5.2320 & \\
\hline
\end{tabular}

As shown, the maximum Von Mises stress for any relative density and any thickness was smaller than the yield strength of the lattice for the corresponding relative density.

\subsubsection{Interface Failure}

To evaluate the interface failure function, normal and shear stresses at bone and implant interface were found using FEA for each individual model. These stresses were used to calculate the interface failure probability by using Equation (4.10) and then the failure probability index in Equation (4.9) was calculated for each model. The results are shown in Table 4.3. 
Table 4.3: Interface failure index results

\begin{tabular}{llllllllll}
\hline & \multicolumn{7}{c}{ Relative Density } \\
\hline Thickness of & 0.12 & 0.15 & 0.20 & 0.25 & 0.30 & 0.35 & 0.40 & 0.45 & 0.50 \\
Lattice(mm) & & & & & & & & & \\
$t=0.2$ & 0.778 & 0.787 & 0.798 & 0.808 & 0.815 & 0.821 & 0.826 & 0.829 & 0.834 \\
$t=0.4$ & 0.768 & 0.775 & 0.785 & 0.794 & 0.801 & 0.808 & 0.814 & 0.820 & 0.823 \\
$t=0.6$ & 0.767 & 0.774 & 0.783 & 0.791 & 0.798 & 0.805 & 0.811 & 0.817 & 0.822 \\
$t=0.8$ & 0.777 & 0.782 & 0.788 & 0.796 & 0.802 & 0.809 & 0.815 & 0.821 & 0.826 \\
$t=1.0$ & 0.785 & 0.788 & 0.794 & 0.801 & 0.807 & 0.813 & 0.819 & 0.825 & 0.830 \\
$t=1.2$ & 0.791 & 0.794 & 0.800 & 0.807 & 0.814 & 0.820 & 0.826 & 0.831 & 0.837
\end{tabular}

\subsubsection{Bone Loss}

As already discussed in Section 4.4, the bone loss can be determined by considering the number of underloaded bone elements around the implant. To investigate the bone loss, the local strain energy of the elements in the presence of the implant were compared to the corresponding local strain energy of the model when there was no implant. Then the bone loss was calculated according to Equation (4.7). These results are tabulated in Error!

\section{Reference source not found..}

Table 4.4 : Bone loss percentage results

\begin{tabular}{|c|c|c|c|c|c|c|c|c|c|}
\hline \multicolumn{10}{|c|}{ Relative density } \\
\hline Thickness of & 0.12 & 0.15 & 0.20 & 0.25 & 0.30 & 0.35 & 0.40 & 0.45 & 0.50 \\
\hline \multicolumn{10}{|l|}{ Lattice (mm) } \\
\hline$t=0.2$ & 10.0 & 10.2 & 8.2 & 7.0 & 6.4 & 6.1 & 5.8 & 5.5 & 5.2 \\
\hline$t=0.4$ & 9.9 & 9.5 & 8.4 & 7.1 & 6.0 & 5.4 & 4.9 & 4.6 & 4.3 \\
\hline$t=0.6$ & 8.2 & 7.8 & 6.7 & 5.9 & 4.8 & 3.9 & 2.9 & 2.5 & 2.0 \\
\hline$t=0.8$ & 5.6 & 5.4 & 4.8 & 3.9 & 2.9 & 1.9 & 1.4 & 0.9 & 0.6 \\
\hline$t=1.0$ & 4.7 & 3.9 & 3.5 & 2.8 & 2.2 & 1.6 & 0.9 & 0.6 & 0.3 \\
\hline$t=1.2$ & 4.0 & 2.9 & 2.4 & 1.8 & 1.4 & 0.9 & 0.5 & 0.3 & 0.2 \\
\hline
\end{tabular}


Figure 4.4 and Figure 4.5 depict the results for interface failure index and bone loss percentage as a function of relative density and thickness of the implant interface made out of lattice. The results of interface failure index in Table 4.3 show that for any given thickness, the interface failure index increased by increasing the relative density. However, by increasing the thickness from $0.2 \mathrm{~mm}$ to $0.6 \mathrm{~mm}$, interface failure index decreased for every relative density. For thicknesses higher than $0.6 \mathrm{~mm}$ the interface failure index increased. Therefore, the minimum value for this function happened at a thickness $0.6 \mathrm{~mm}$ and relative density of 0.12 as can be seen in Figure 4.4. This figure shows how the interface failure function changes by varying the relative density and thickness of the lattice zone. At possible minimum interface failure, the mass reduction would be $29 \%$ in comparison to a solid implant model. According to Table 4.4, the minimum bone loss happened when the interface zone thickness was $1.2 \mathrm{~mm}$ and relative density was 0.5 . This can be also seen in Figure 4.5. At this point, bone loss is $0.2 \%$ and hence it can be selected as the optimum point when only bone loss is the objective. At this point, the mass reduction would be $27 \%$ in comparison to a solid implant. 


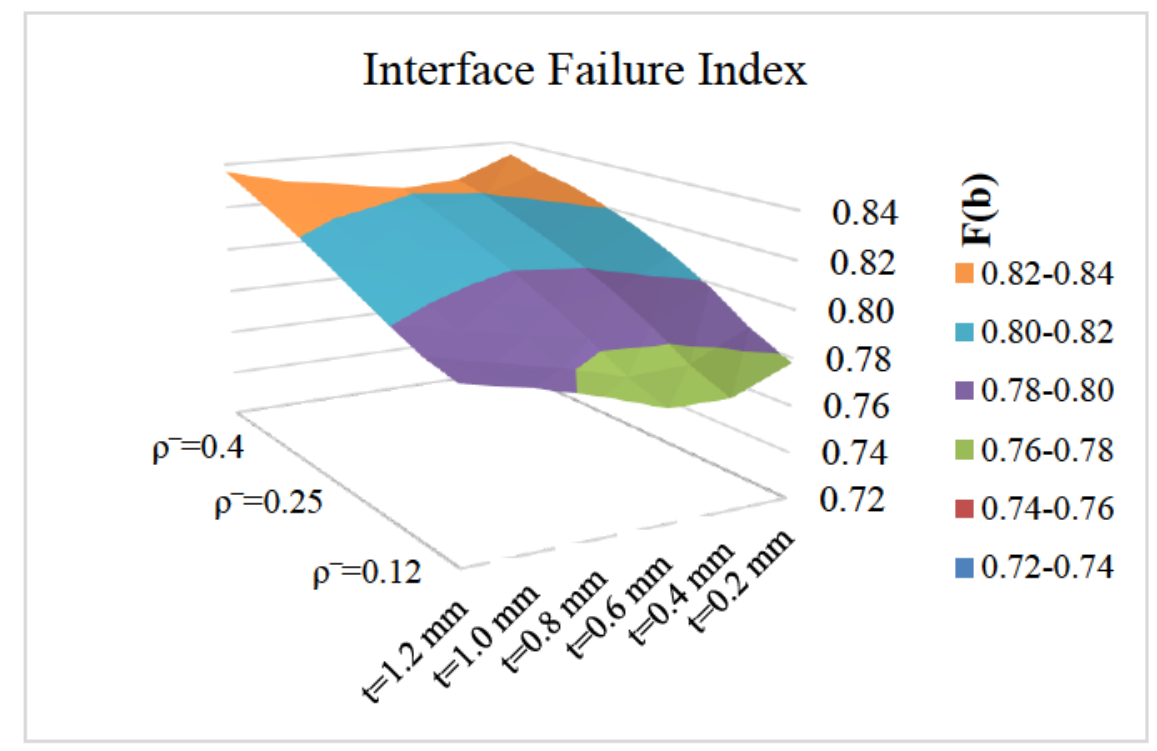

Figure 4.4: The effect of relative density and implant interface thickness at interface failure index.

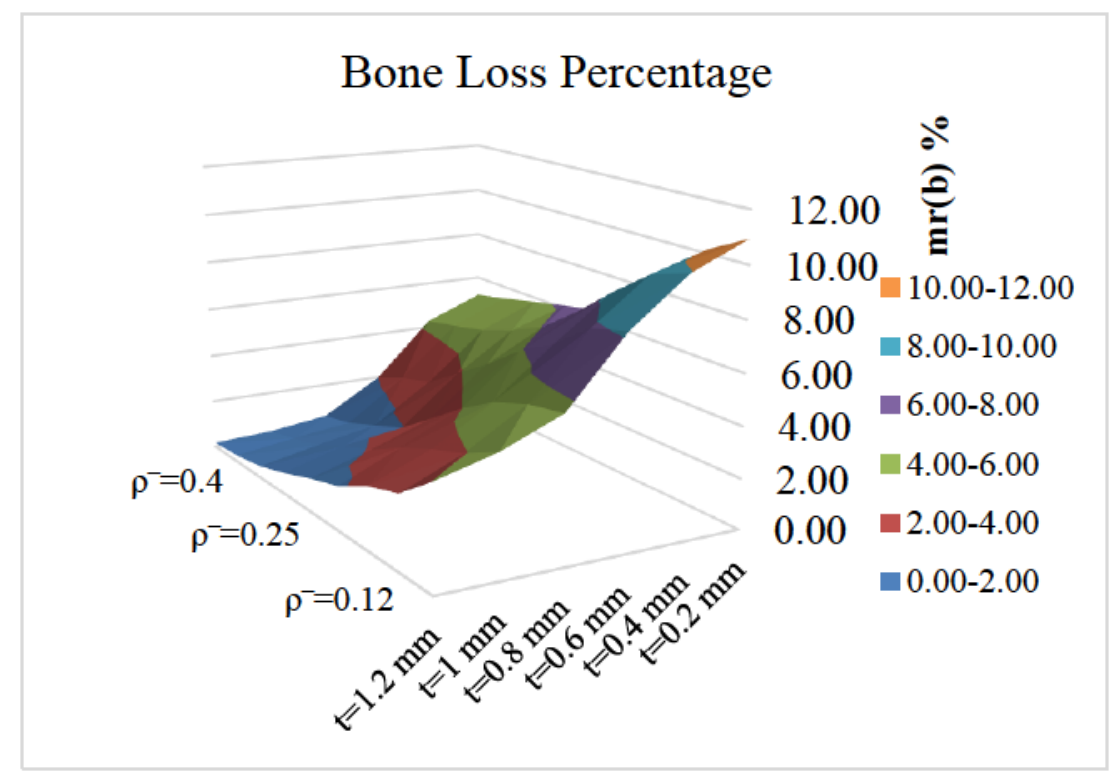

Figure 4.5: The effect of relative density and implant interface thickness at bone loss percentage 


\subsubsection{Pareto Optimal Solutions}

In multiobjective optimizations, there is no unique solution that minimizes all objective functions simultaneously. Instead, there is a set of optimum solutions, called Pareto Optimal solutions that does not require the designer to choose in advance any weighting factors to the objective functions. When a set of all available solutions has been determined, one may easily choose the desired solutions based on the importance of the objective functions [47]. Figure 4.6 depicts all available solutions of multiobjective optimization of dental implant. The relative density of the lattice changes between $0.12-0.5$ and lattice interface zone thickness changes between $0.2-1.2 \mathrm{~mm}$. The $x$ axis of this plot represents the bone loss percentage and the $y$ axis is the global interface failure function. Six different available optimal solutions are marked in this figure. Solution $\mathrm{A}$ is the case when the lattice zone thickness is $1.2 \mathrm{~mm}$ with $50 \%$ relative density and this design provides the minimum bone loss, while the interface failure reaches its maximum value. Solutions B, C and D can be considered as optimum designs that can simultaneously reduce bone loss and interface failure. By taking into account the mass reduction, design B reduces weight by $46 \%$ and by $41 \%$ and $43 \%$, respectively, for designs $\mathrm{C}$ and $\mathrm{D}$. 


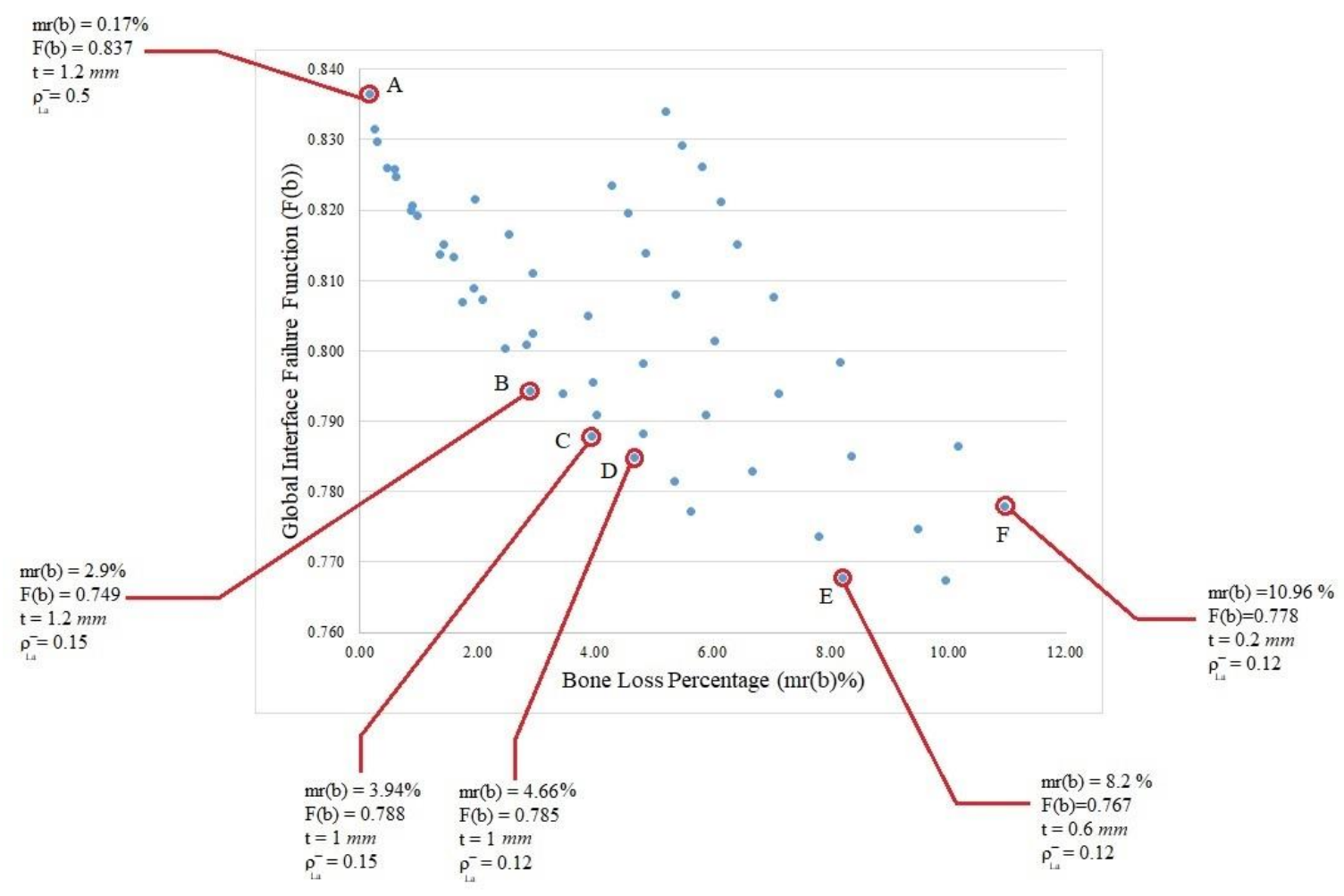

Figure 4.6: Pareto optimal solutions of bone loss and interface failure

Design E represents a dental implant with $0.6 \mathrm{~mm}$ thickness in lattice zone with $12 \%$ relative density. This structure of implant causes the minimum interface failure on boneimplant interface. Moving from point $\mathrm{E}$ to point $\mathrm{F}$, bone loss increases and reaches its maximum.

In Figure 4.7 the Pareto optimal solutions ( those that are not dominated by any other feasible solutions) are marked by a red curve. Any point on this curve can be considered as a Pareto frontier solution and can be selected to design the implant. 


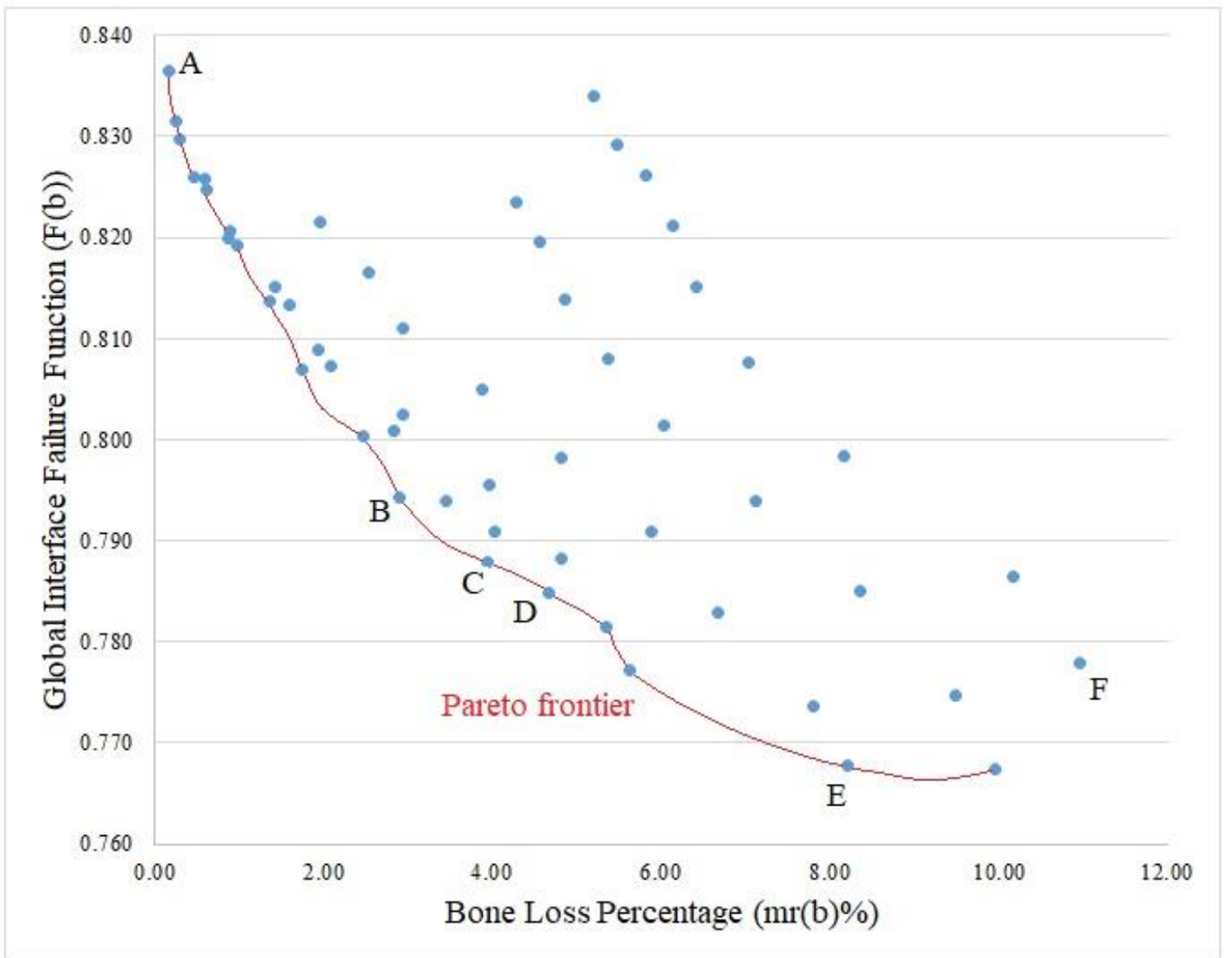

Figure 4.7: Pareto frontier solutions of bone loss and interface failure

\subsubsection{Stress Distribution in Jaw Bone for Solid and Lattice Implants}

The stress distribution of bone when a solid implant is inserted into the jawbone are shown in Figure 4.8 and Figure 4.9. As shown, the maximum stresses in cancellous bone and cortical bone are $0.867 \mathrm{MPa}$ and $10.81 \mathrm{MPa}$, respectively. Figure 4.10 and Figure 4.11 illustrate the stress distribution for cancellous bone and cortical bone, when a lattice implant of type $\mathrm{E}$ is inserted into the jaw bone. This type of implant could provide the minimum interface failure and as can be seen, the maximum stress in cancellous bone and cortical bone are 1.093 $\mathrm{MPa}$ and 22.04 $\mathrm{MPa}$ respectively which are higher than stresses in 
the bone when a solid implant was used. Using lattice material in implant structure creates an interface with a lower Young's modules than the solid implant and it allows the stresses to be transferred better and smoother to the surrounding bone.

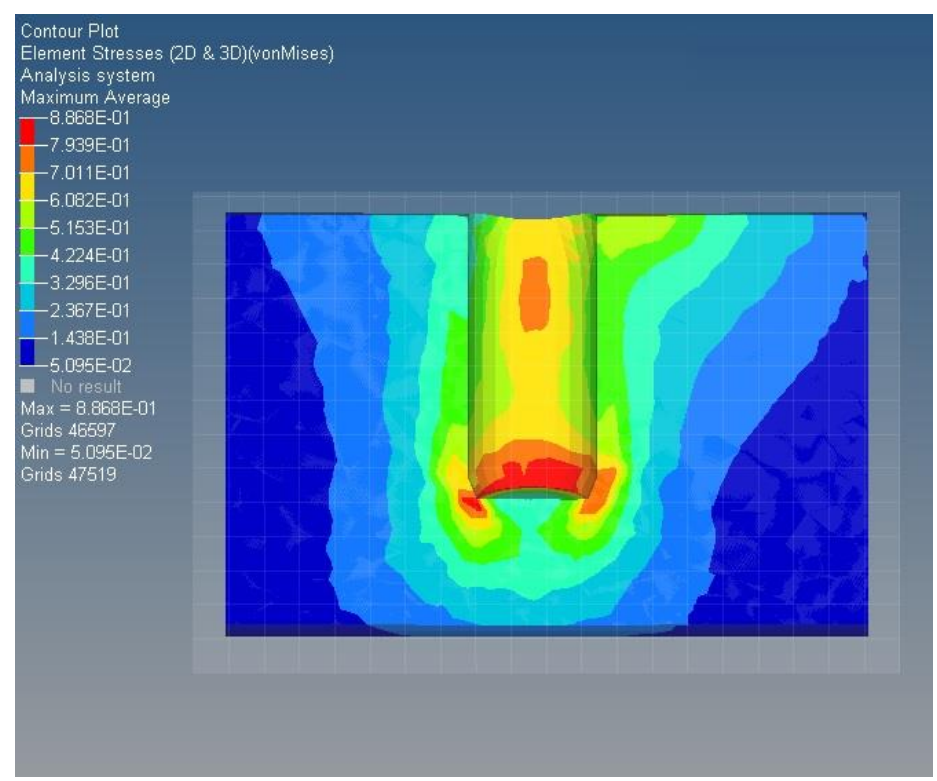

Figure 4.8: Von Mises stress distribution in cancellous bone in presence of a solid implant

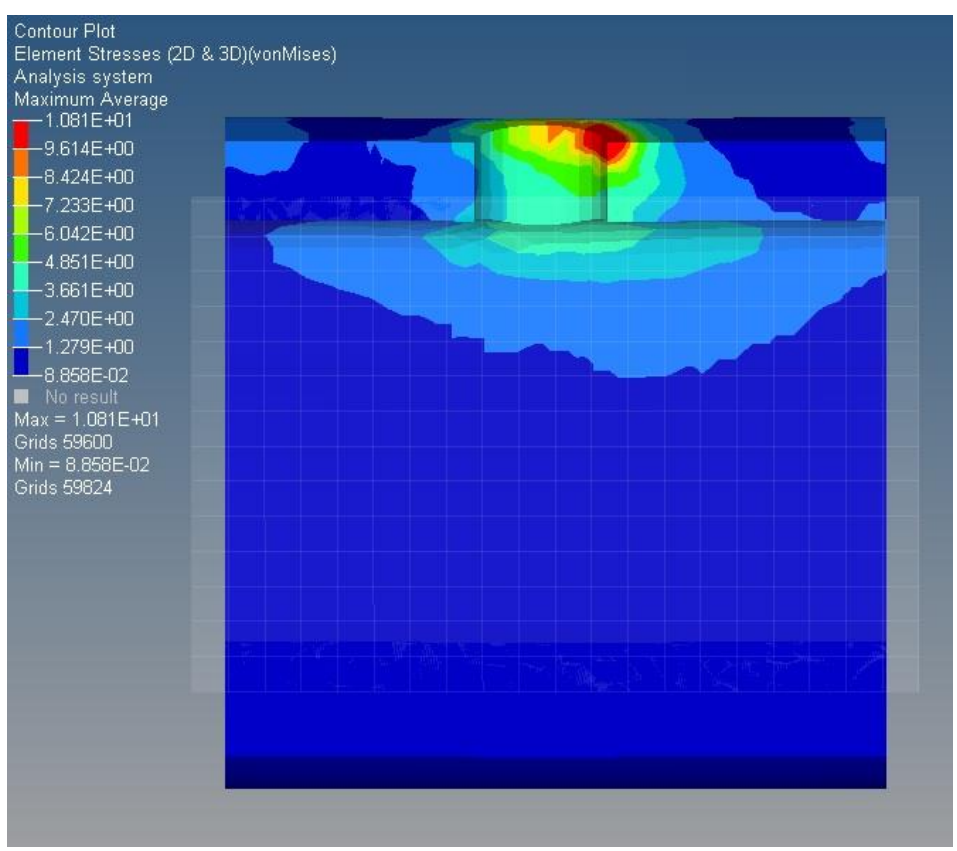

Figure 4.9: Von Mises stress distribution in cortical bone in presence of a solid implant 


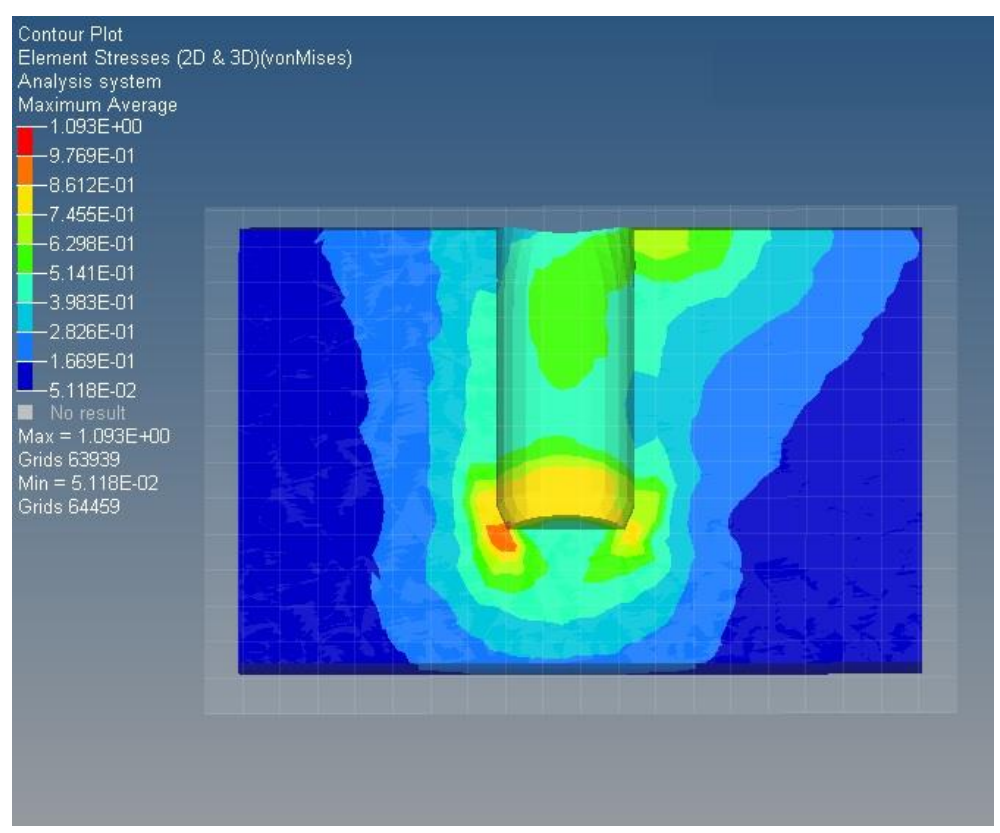

Figure 4.10: Von Mises stress distribution in cancellous bone in presence of implant typeE

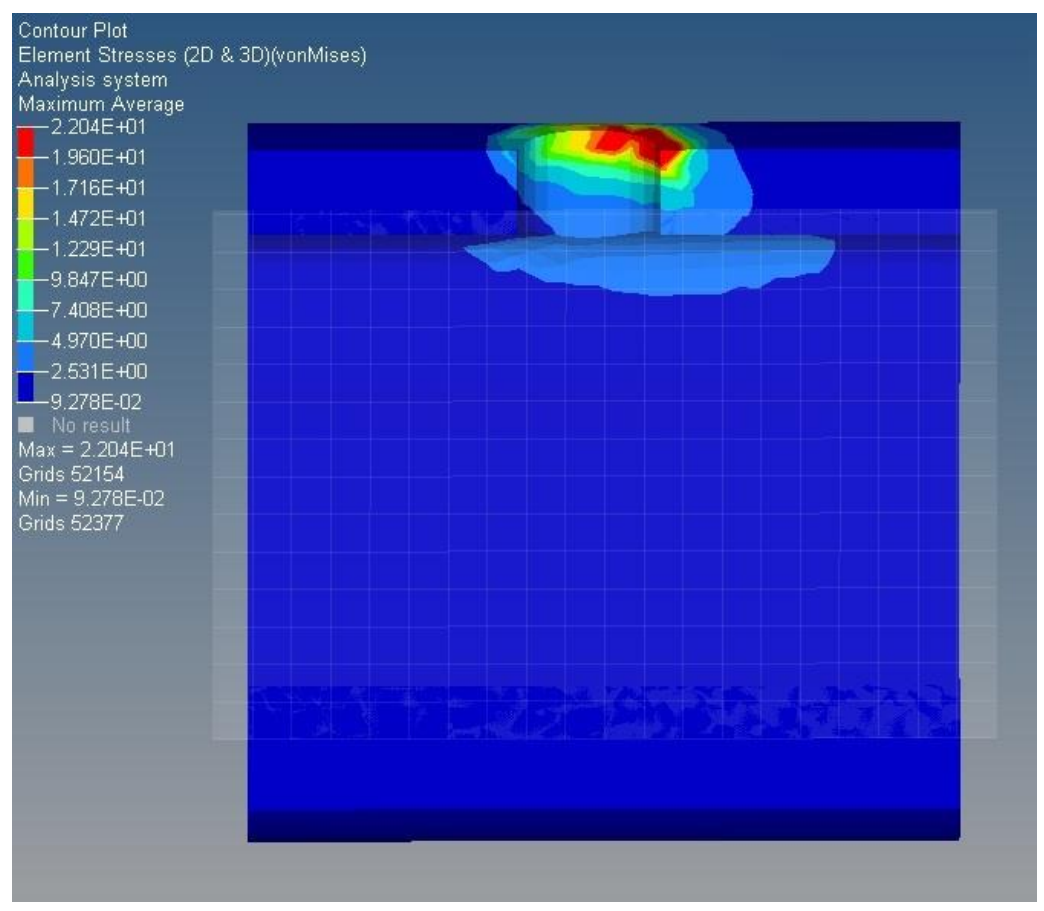

Figure 4.11: Von Mises stress distribution in cortical bone in presence of implant type E 


\subsubsection{Maximum Von Mises Stress in Jaw Bone for Solid and Lattice Implants}

As discussed in the previous section the FEA results showed that the minimum bone loss happened in an implant with $t=1.2 \mathrm{~mm}$ thickness and relative density of $50 \%$. The minimum interface failure occurred in an implant with lattice zone thickness of $0.6 \mathrm{~mm}$ and relative density of $12 \%$. Also designs B, C and D on Figure 4.7 were presented as the optimum solutions that can simultaneously reduce the bone loss and interface failure. In this section the stress distribution in bone for optimum implants and a whole solid implant are discussed. As shown in Figure 4.12, the maximum Von-Mises stress in the jaw bone, when a solid implant has been used, is $10.81 \mathrm{MPa}$, while for implant type (A) $\left(t=1.2 \mathrm{~mm}, \bar{\rho}_{L a}=0.5\right)$, which is the optimum design for minimum bone loss, the Von Mises stress is $21.83 \mathrm{MPa}$ as shown in Figure 4.13. This suggests that the lattice structure in implant design would increase the stress in bone and decrease the bone loss.

The stress distribution in bone for lattice implant type (C) $\left(t=1 \mathrm{~mm}, \bar{\rho}_{L a}=0.15\right)$ is depicted in Figure 4.14 and shows the maximum Von Mises stress is 25.19 MPa and Figure 4.15 represents the stress distribution of lattice implant type $\mathrm{E}\left(t=0.6 \mathrm{~mm}, \bar{\rho}_{L a}=0.12\right)$ with the maximum Von Mises stress of 22.04 MPa.

Overall, it can be seen that the stress distribution in bone is strongly dependent on the structure of the implants. Under the same loading, the stress in bone is higher when a lattice implant is inserted inside the bone. In fact, the lattice structure in the implant body, helps the interface stress can be transferred better to the surrounding bones, avoiding stress shielding and concentration. 


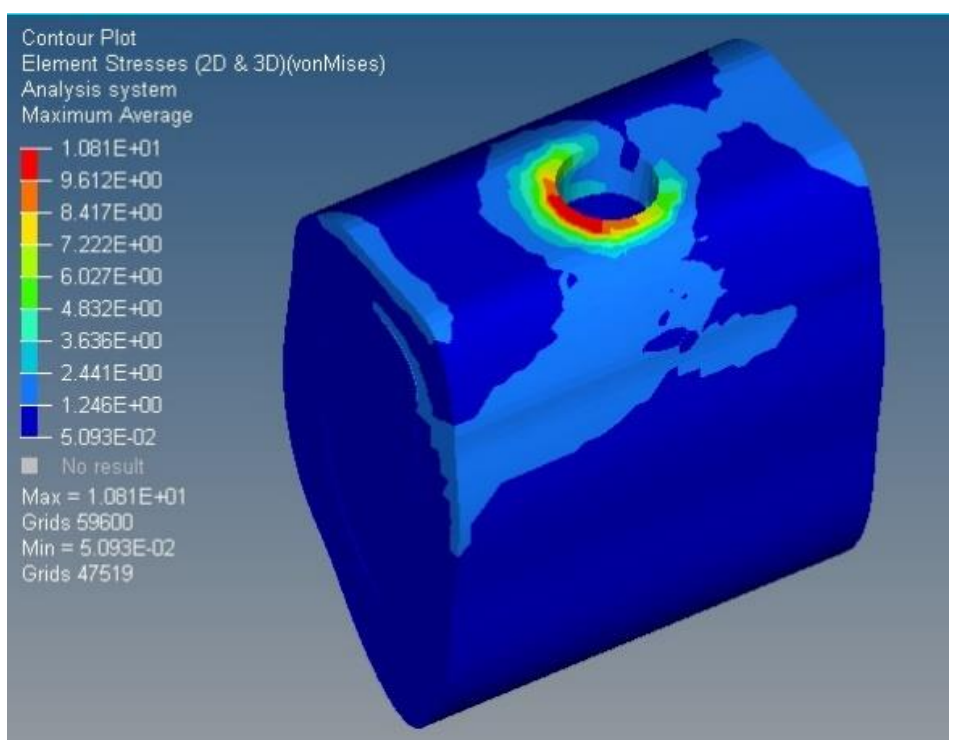

Figure 4.12: Von-Mises stress distribution in bone with solid implant

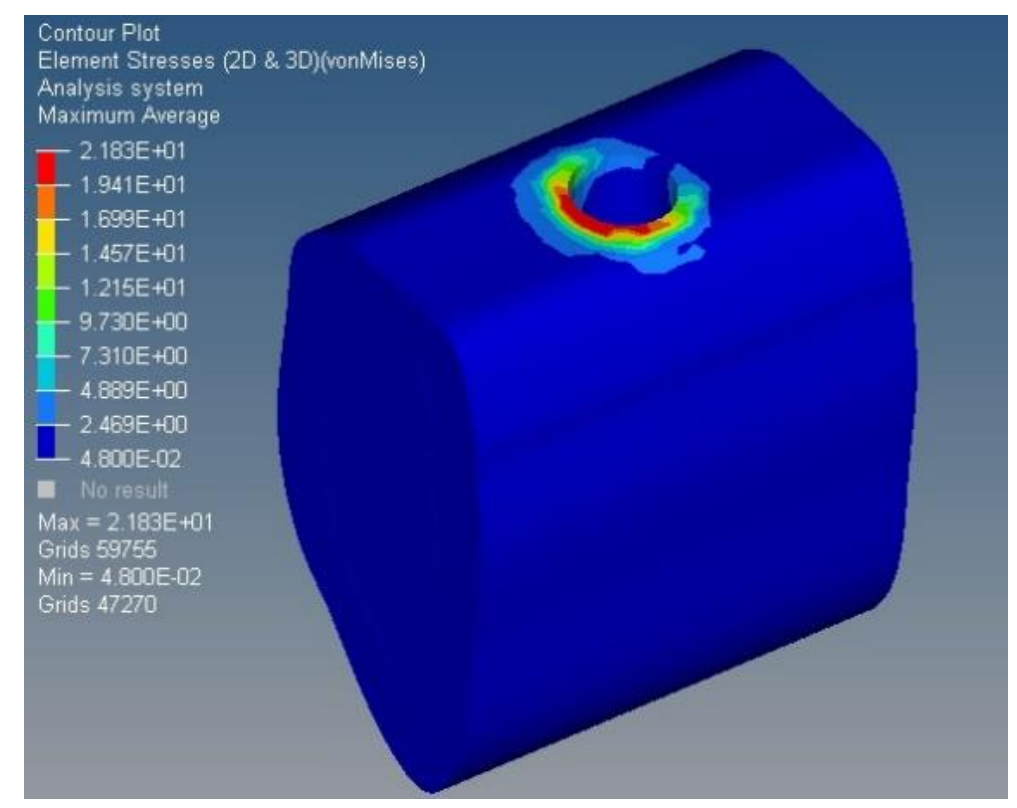

Figure 4.13: Von-Mises stress distribution in bone with lattice implant type (A), 


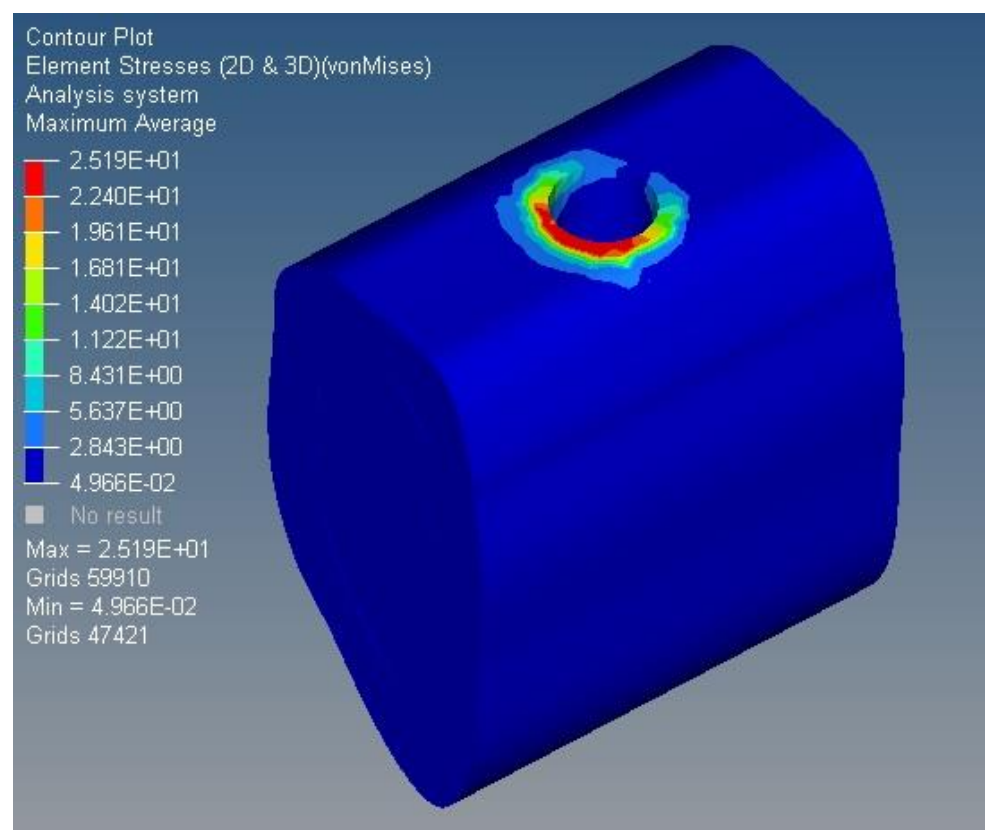

Figure 4.14: Von-Mises stress distribution in bone with lattice implant type (C)

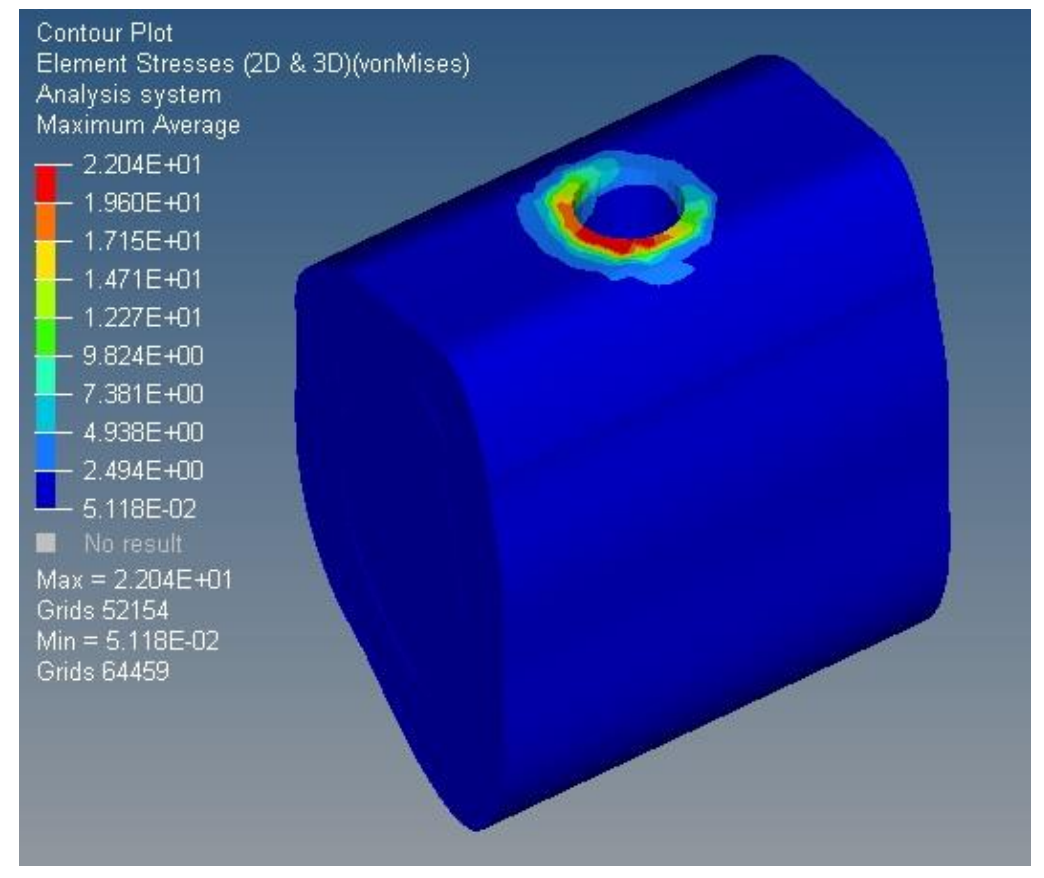

Figure 4.15: Von-Mises stress distribution in bone with lattice implant type (E) 
Figure 4.16 shows the implant structure with minimum bone loss expectation with an interface zone thickness of $1.2 \mathrm{~mm}$ and a relative lattice density of 0.5 that can be considered as one of the optimum designs of the dental Implant.

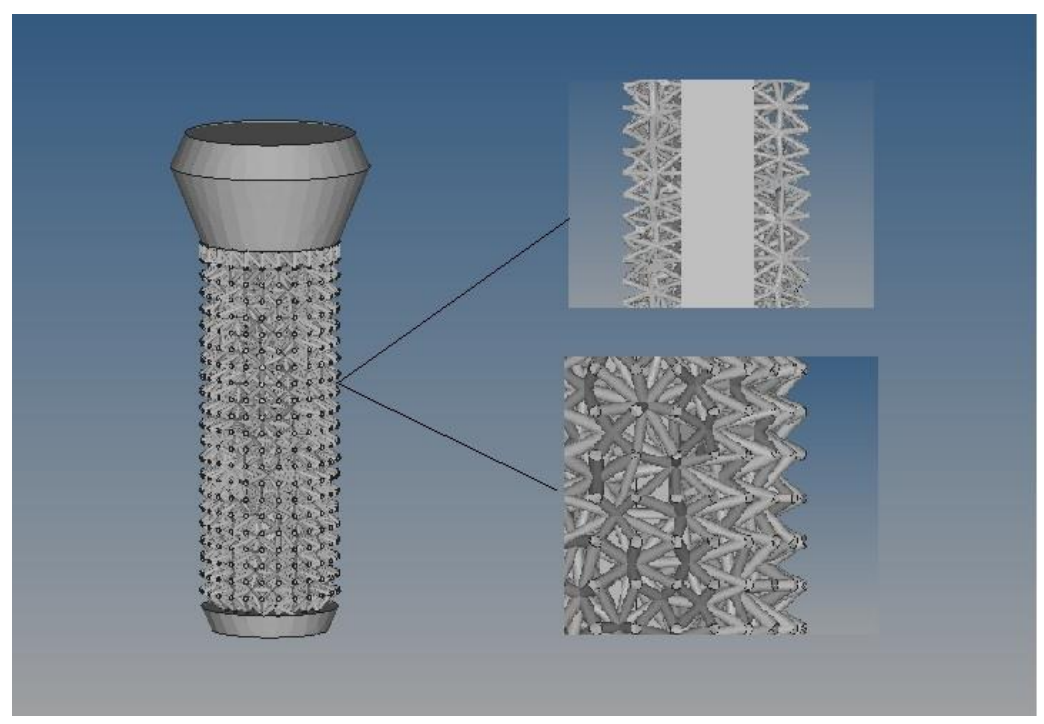

Figure 4.16: Implant structure after optimization

\subsection{Discussion}

In this chapter, Finite Element Analysis was used to evaluate the effect of lattice structure in implant interface. The implant was considered as a cylinder-shaped model without the threads. The implant is made out of a solid core in cylindrical part and lattice part in interface zone. The octet-truss lattice was selected as the type of lattice unit cell and the interface thickness and lattice unit cell relative density were the design variables. Then the static stress analysis was performed to determine the normal and shear stresses at bone implant interface. The results then were used to find the local interface failure probability as well as the global interface failure functions. The results showed that by increasing the interface thickness from $0.2 \mathrm{~mm}$ to $0.6 \mathrm{~mm}$ the interface failure decreases. But by 
increasing the thickness from $0.6 \mathrm{~mm}$ to $2 \mathrm{~mm}$, the interface failure index increases. So in order to have a minimum interface failure index the optimum thickness would be $0.6 \mathrm{~mm}$ for an implant with a diameter of $4.1 \mathrm{~mm}$ in the cylindrical part. To evaluate the bone loss, the strain energy densities of interface bone elements were studied and the results showed that by increasing the thickness and the relative density of the lattice part, the bone loss is decreasing and the minimum bone loss happens at thickness of $1.2 \mathrm{~mm}$ and $\% 50$ of relative density. The simulation results showed that, the interface failure function and bone loos are competing to each other. Where the minimum bone loss happens, the interface failure reaches to its maximum. This result was also obtained in the work of hip implant optimization by Arabnejad and Pasini [53] Their results also showed that by reducing the bone loss interface failure increases and vise versa. In order to reduce the bone loss and interface failure simultaneously, the optimum design can be selected from the Pareto frontier points in Figure 4.7 such as designs B, C or D. Using the lattice structure in implant design not only reduces the interface failure and the bone loss, it can also make the bone growth easier for the jaw bone. It helps the stresses to be transferred to the surrounding bone. In comparison to solid implants, a lattice structure provides more space for bone to grow. So it can decrease the bone loss around the implant. Also the optimum designs can reduce the weight by $27-46 \%$. 


\section{Conclusions and Future Work}

\subsection{Summary}

As previously discussed, dental implants are artificial tooth replacements that are made, mainly, out of solid metals and used to serve as an artificial dental tooth to support the crowns. Although, the rate of the success in most of the implants is quite high in terms of long term survival and mechanical strength, there are still some problems existing about them including bone-implant interface failure and bone loss. These two culprits mainly happen because of the stiffness mismatch between the implant and bone. Current dental implants are usually made out of high stiffness materials like titanium while the stiffness of the bone is very small in compare with the dental implant. This stiffness mismatching prevents the stresses to be transferred to the surrounding bone and causes bone resorption and interface failure. In this work, design and optimization of a cylindrical dental implant, using additively manufactured lattice materials has been considered as one solution to overcome the bone loss and interface failure. Lattice materials are cellular periodic structures that can be generated by tessellating a unit cell. The main purpose of using this material in any design, is reducing the weight, increasing the efficiency and using as little material as possible. A very important feature of lattice materials is that they can be designed for any required mechanical properties. Since the stiffness mismatching is a problem in current dental implants, lattice materials can be used in the implant design to eliminate or reduce this stiffness mismatching. In Chapter 3 lattice materials, their classification and their effective mechanical properties were discussed and octet-truss lattice unit cell was selected to use in dental implant design and optimization. In Chapter 4, topology optimization was considered as a possible method to perform the optimization. 
Finite element software, Altari OptiStruct was used to study and demonstrate some basic optimization problems. In Chapter 5 multiscale design optimization of dental implant was studied. A 3D model of a segment of a jawbone and a cylindrical dental implant was developed. The implant was considered as a cylinder-shaped model without the threads. The implant was assumed to be made out of a solid core in cylindrical part and octet-truss lattice part in interface zone. The interface thickness and lattice unit cell relative density were chosen to be the design variables. Optimization problem was formulated and finite element analysis was used to evaluate the objective functions. The results showed that the minimum amount of interface failure can happen at the thickness of $0.6 \mathrm{~mm}$ and the relative density of $12 \%$. While the minimum bone loss happens at the thickness of $1.2 \mathrm{~mm}$ and the relative density of $50 \%$. To simultaneously minimize these functions, Pareto frontier curve can be used to design the implant. Finite element analysis of some of the optimal solutions showed that the use of lattice materials in implant structure, not only reduces the interface failure and bone loss, it can also increase the maximum Von Mises stress in bone in comparison to solid implants which means the stiffness mismatching between the bone and implant is reduced. In this work design and optimization of dental implant was mostly studied based on the defined objective functions.

\subsection{Future Work}

Design and optimization of a cylindrical dental implant was studied in this work using additive manufactured lattice materials. There are many other different types of implants including different dental implants as explained in Chapter 2 of this work, that can be considered for optimization with lattice materials. In this study, only one type of lattice 
unit cell was selected to design the implant, while other types of lattice unit cells can also be considered to perform the optimization problem. Also, the final optimized model of the implant can be printed by 3D printers and be used for some experimental works. In this work, the static analysis of dental implants was considered for implant design and optimization, while in the future work, fatigue analysis of the implants can be studied and considered for a more precise design. In vivo studies can also be considered for some experimental works such as evaluating the effect of implant porous structure on bone loss and implant and bone biomechanical bonding. The manufacturability of the presented implant was not a part of this work but it can be considered in the future works to evaluate the feasibility of the design and any further optimization. In this work the interface between lattice zone and bone was studied, but in the future works, the interface between solid core and lattice zone can be evaluated to perform optimization. Automation of the optimization process by using different softwares can be implemented in the future works to include more design variables in the work. In addition, the automation will help to build the patient specific implant based on their bone density distribution and their jaw bone sizes. 


\section{References}

[1] Van Staden RC, Guan H, Loo YC: Application of the finite element method in dental implant research. Comput Methods Biomech Biomed Engin 2006;9:25770.doi:10.1080/10255840600837074.

[2] Pektas O: Design and mechanical analysis of a new dental implant that would mimic natural tooth with a periodontal ligament 2012.

[3] O’Brien W.J: Dental Mateirals: Properties and Selection. Chicago; London: Quintessence Publishing; 1989.

[4] Geng J, Xu W, Yan W: Model-based biomechanical dental implant optimization in bone- implant system 2007:15-22.

[5] Shi L, Li H, Fok ASL, Ucer C, Devlin H, Horner K: Shape optimization of dental implants. Int J Oral Maxillofac Implant 2007;22.

[6] Khanoki SA: Multiscale mechanics and multiobjective optimization of cellular hip implants with variable stiffness 2013.

[7] Wauthle R, Van Der Stok J, Yavari SA, Van Humbeeck J, Kruth JP, Zadpoor AA, et al.: Additively manufactured porous tantalum implants. Acta Biomater 2015;14:217-25.doi:10.1016/j.actbio.2014.12.003.

[8] Wally Z, van Grunsven W, Claeyssens F, Goodall R, Reilly G: Porous Titanium for Dental Implant Applications. Metals (Basel) 2015;5:1902-20.doi: 10.3390/ met 5041902.

[9] Chahine G, Atharifar H, Smith P: Design Optimization of a Customized Dental Implant Manufactured via Electron Beam Melting. Int Solid Free Fabr Symp 2009:631-40. 
[10] Sadollah A, Bahreininejad A: Optimum gradient material for a functionally graded dental implant using metaheuristic algorithms. J Mech Behav Biomed Mater 2011;4:1384-95.doi:10.1016/j.jmbbm.2011.05.009.

[11] Jamshidinia M, Wang L, Tong W, Kovacevic R: The bio-compatible dental implant designed by using non-stochastic porosity produced by Electron Beam Melting. J Mater Process Technol 2014;214:1728-39.doi:10.1016/j.jmatprotec.2014.02.025.

[12] Albrektsson T: The long-term efficacy of currently used dental implants: Areview and proposed criteria of success 1997.

[13] Geng JP, Tan KB, Liu GR: Application of finite element analysis in implant dentistry: a review of the literature. J Prosthet Dent 2001;85:585-98.doi:10.1067/ mpr.2001.115251.

[14] Brunski JB: In vivo Bone Response to Biomechanical Loading at the Bone/DentalImplant Interface 1999:99-119.

[15] Pilliar RM1, Deporter DA, Watson PA VN: Dental implant design--effect on bone remodeling. J Biomed Mater Res 1991;25:467-83.

[16] Clift SE, Fisher J, Watson CJ: Stress and strain distribution in the bone surrounding a new design of dental implant: a comparison with a threaded Branemark type implant. Proc Inst Mech Eng H 1993;207:133-8.doi:10.1243/ PIME_PROC_ 1993_207_285_02.

[17] Vidyasagar L, Apse P: Dental Implant Design and Biological Effects on BoneImplant Interface 2004;6:51-4.

[18] Osman R, Swain M: A Critical Review of Dental Implant Materials with an Emphasis on Titanium versus Zirconia. Materials (Basel) 2015;8:932-58.doi: 
10.3390/ma8030932.

[19] Junker R, Dimakis A, Thoneick M, Jansen JA: Effects of implant surface coatings and composition on bone integration: A systematic review. Clin Oral Implants Res 2009;20:185-206.doi:10.1111/j.1600-0501.2009.01777.x.

[20] Lefebvre LP, Banhart J, Dunand DC: Porous metals and metallic foams: Current status and recent developments. Adv Eng Mater 2008;10:775-87.doi:10.1002/ adem.200800241.

[21] Mour, M.; Das, D.; Winkler, T.; Hoenig, E.; Mielke, G.; Morlock, M.M.; Schilling AF: Advances in Porous Biomaterials for Dental and Orthopaedic Applications. Materials 2010;3:2947-2974.

[22] Li X, Wang C, Zhang W, Li Y: Fabrication and compressive properties of Ti6Al4V implant with honeycomb-like structure for biomedical applications: Rapid Prototyp J 2010;16:44-9.doi:10.1108/13552541011011703.

[23] Grunsven W Van: Porous metal implants for enhanced bone ingrowth and stability Thesis submitted to the University of Sheffield for the degree of Doctor of Philosophy. 2014.

[24] Ahmadi SM, Campoli G, Amin Yavari S, Sajadi B, Wauthle R, Schrooten J, et al.: Mechanical behavior of regular open-cell porous biomaterials made of diamond lattice unit cells. J Mech Behav Biomed Mater 2014;34:106-15.doi:10.1016/j. jmbbm.2014.02.003.

[25] Otsuki B, Takemoto M, Fujibayashi S, Neo M, Kokubo T, Nakamura T: Pore throat size and connectivity determine bone and tissue ingrowth into porous implants: Three-dimensional micro-CT based structural analyses of porous bioactive titanium 
implants. Biomaterials 2006;27:5892-900.doi:10.1016/j.biomaterials.2006.08.013.

[26] Goodall, R.; Mortensen A: Porous Metals. In Physical Metallurgy. 5th ed. Amsterdam, The Netherlands: Elsevier; 2014.

[27] Gibson L., Ashby MF: Cellular solids structure and properties. Cambridge, UK: Cambridge University Press; 1999.

[28] Tao W, Leu MC: Design of lattice structures for additive manufacturing. 2016 Int Symp Flex Autom 2016:1-3.

[29] McDowell A-JW and DL: In-Plane Stiffness and Yield Strength of Periodic Metal Honeycombs. J Eng Mater Technol 2004;126:137-56.

[30] Wang AJ, McDowell DL: Yield surfaces of various periodic metal honeycombs at intermediate relative density. Int J Plast 2005;21:285-320.doi:10.1016/ j.ijplas. 2003.12.002.

[31] Cheng GD, Cai YW, Xu L: Novel implementation of homogenization method to predict effective properties of periodic materials. Acta Mech Sin Xuebao 2013;29:550-6.doi:10.1007/s10409-013-0043-0.

[32] Arabnejad S, Pasini D: Mechanical properties of lattice materials via asymptotic homogenization and comparison with alternative homogenization methods. Int $\mathrm{J}$ Mech Sci 2013;77:249-62.doi:10.1016/j.jjmecsci.2013.10.003.

[33] Hutchinson RG, Fleck NA: The structural performance of the periodic truss. J Mech Phys Solids 2006;54:756-82.doi:10.1016/j.jmps.2005.10.008.

[34] Elsayed MSA, Pasini D: Analysis of the elastostatic specific stiffness of 2D stretching-dominated lattice materials. Mech Mater 2010;42:709-25.doi:10.1016/ j.mechmat.2010.05.003. 
[35] Elsayed MSA, Pasini D: Multiscale structural design of columns made of regular octet-truss lattice material. Int J Solids Struct 2010;47:1764-74.doi:10.1016/ j.ijsolstr.2010.03.003.

[36] Huang Y, Xue Y, Wang X, Han F: Mechanical behavior of three-dimensional pyramidal aluminum lattice materials. Mater Sci Eng A 2017;696:520-8.doi: 10.1016/j.msea.2017.04.053.

[37] Messner MC: Optimal lattice-structured materials. J Mech Phys Solids 2016;96: 162-83.doi:10.1016/j.jmps.2016.07.010.

[38] Mancusi G, Fabbrocino F, Feo L, Fraternali F: Size effect and dynamic properties of 2D lattice materials. Compos Part B Eng 2016;112:235-42.doi:10.1016/ j.compositesb.2016.12.026.

[39] Vigliotti A, Deshpande VS, Pasini D: Non linear constitutive models for lattice materials. J Mech Phys Solids 2014;64:44-60.doi:10.1016/j.jmps.2013.10.015.

[40] Liu H, Zhang HW: An equivalent multiscale method for 2D static and dynamic analyses of lattice truss materials. Adv Eng Softw 2014;75:14-29.doi: 10.1016/ j.advengsoft.2014.04.006.

[41] Vigliotti A, Pasini D: Mechanical properties of hierarchical lattices. Mech Mater 2013;62:32-43.doi:10.1016/j.mechmat.2013.03.003.

[42] Ashby MF: The properties of foams and lattices. Philos Trans A Math Phys Eng Sci 2006;364:15-30.doi:10.1098/rsta.2005.1678.

[43] Kuiper JH, Huiskes HWJ: Numerical optimization of hip-prosthetic stem material 1992.

[44] Shi L, Li H, Fok ASL, Ucer C, Devlin H, Horner K: Shape optimization of dental 
implants. Int J Oral Maxillofac Implant 2007;22.

[45] Lodygowski T, Szajek K, Wierszycki M: Optimization of dental implant using genetic algorithm. J Theor Appl Mech 2009;47:573-98.

[46] Lin D, Li Q, Li W, Zhou S, Swain M V.: Design optimization of functionally graded dental implant for bone remodeling. Compos Part B Eng 2009;40:668-75.doi: 10.1016/ j.compositesb.2009.04.015.

[47] Arabnejad, K S, Pasini D: Multiscale Design and Multiobjective Optimization of Orthopedic Hip Implants with Functionally Graded Cellular Material. J Biomech Eng 2012;134:031004.doi:10.1115/1.4006115.

[48] Chang C-L, Chen C-S, Huang C-H, Hsu M-L: Finite element analysis of the dental implant using a topology optimization method. Med Eng Phys 2012;34:9991008.doi:10.1016/j.medengphy.2012.06.004.

[49] Magusiak M\& B: Implant Dentistry. Http://WwwMmbdentistryCom 2018. http://www.mmbdentistry.com/specialty-treatments/implant-dentistry/.

[50] Classification of Dental Implants. Http://Solid-TipsCom/2014/04/ClassificationDental-Implants/2014.http://solid-tips.com/2014/04/classification-dental-implants/.

[51] Baby Sowdeswari.R, M. Benzir Begam, S. Birundha, M.Brundha RD: Dental Implants types and clasification. 2011.

[52] Noorthoek DR: Macroscopic and microscopic dental implant design: A review of the literature 2013.

[53] Arabnejad Khanoki S, Pasini D: Multiscale Design and Multiobjective Optimization of Orthopedic Hip Implants with Functionally Graded Cellular Material. J Biomech Eng 2012;134:031004.doi:10.1115/1.4006115. 
[54] Ojeda J, Martinez-Reina J, Garcia-Aznar JM, Dominguez J, Doblare M: Numerical simulation of bone remodelling around dental implants. Proc Inst Mech Eng Part HJournal Eng Med 2011;225:897-906.doi:10.1177/0954411911410165.

[55] Elsayed MS a: Multiscale Mechanics and Structural Design of Periodic Cellular Materials 2010:308.doi:10.1016/j.msec.2009.10.004.

[56] Tian H, Tice J, Fei R, Tran V, Yan X, Yang L, et al.: Low-symmetry twodimensional materials for electronic and photonic applications. Nano Today 2016;11:1-37.doi:10.1016/j.nantod.2016.10.003.

[57] Chopra P: Effective Mechanical Properties of Lattice Materials 2011.

[58] Elsayed MSA, Pasini D: Multiscale structural design of columns made of regular octet-truss lattice material. Int J Solids Struct 2010;47:1764-74.doi:10.1016/ j.ijsolstr.2010.03.003.

[59] John D. REnton: Elastic Beams and Frames. Woodhead Publishing Limited; 2002.

[60] Browne P a: Topology Optimization of Linear Elastic Structures. 2013.

[61] Cazacu R, Grama L: Overview of Structural Topology Optimization Methods for Plane and Solid Structures. Ann Univ Oradea 2014:17-22.

[62] Rozvany GIN: Aims, scope, methods, history and unified terminology of computeraided topology optimization in structural mechanics. Struct Multidiscip Optim 2001;21:90-108.doi:10.1007/s001580050174.

[63] A.G.M. Michell. LVIII.: The limits of economy of material in frame-structures. London, Edinburgh, Dublin Philos Mag J Sci- Ence, 1904;8:589-597,.

[64] Prager, W.; Rozvany GI.: Optimization of structural geometry. Dyn Syst New York Acad Press 1977:265-293. 
[65] Rozvany, G.I.N.; Zhou M: Applications of COC method in layout optimization. Springer-Verlag 1991:59-70.

[66] Bends??e MP, Kikuchi N: Generating optimal topologies in structural design using a homogenization method. Comput Methods Appl Mech Eng 1988;71:197224.doi:10.1016/0045-7825(88)90086-2.

[67] Bends e MP, Kikuchi N: Generating Optimal Topologies in Structural Design Using a Homogenization Method. Comput Methods Appl Mech Eng 1988;71:197224.

[68] Bendsøe MP: Optimal shape design as a material distribution problem. Struct Optim 1989;1:193-202.doi:10.1007/BF01650949.

[69] Bendsøe MP, Sigmund O: Topology optimization: theory, methods, and applications. vol. 2nd Editio. 2003.doi:10.1063/1.3278595.

[70] Holmberg E: Stress and fatigue constrained topology optimization. vol. 48. 2013.

[71] Altair Engineering, “ Altair OptiStruct,” Altair Engineering, [Online]. 2017.

[72] Brackett D, Ashcroft I, Hague R: Topology optimization for additive manufacturing. Solid Free. Fabr. Symp., 2011, p. 348-62.doi:10.1017/CBO9781107415324.004.

[73] Christensen RM: Mechanics of low density materials. J Mech Phys Solids 1986;34:563-78.doi:10.1016/0022-5096(86)90037-2.

[74] Vigliotti A, Pasini D: Linear multiscale analysis and finite element validation of stretching and bending dominated lattice materials. Mech Mater 2012;46:5768.doi:10.1016/j.mechmat.2011.11.009.

[75] Chen L, He H, Li Y, Li T, Guo X, Wang R: Finite element analysis of stress at implant-bone interface of dental implants with different structures. Trans 
Nonferrous Met Soc China 2011;21:1602-10.doi:10.1016/S1003-6326(11)60903-5.

[76] Mericske-Stern R, Piotti M, Sirtes G: 3-D in vivo force measurements on mandibular implants supporting overdentures. A comparative study. Clin Oral Implants Res 1996;7:387-96.doi:10.1034/j.1600-0501.1996.070413.x.

[77] Kayabaşı O, Yüzbasığlu E, Erzincanlı F: Static, dynamic and fatigue behaviors of dental implant using finite element method. Adv Eng Softw 2006;37:64958.doi:10.1016/j.advengsoft.2006.02.004.

[78] Lower Mandible. VikingDigital 2014.https://www.turbosquid.com/3d-models/3dmandible-model/790899.

[79] Kumar GA, Kovoor LC, Oommen VM: Three-dimensional finite element analysis of the stress distribution around the implant and tooth in tooth implant-supported fixed prosthesis designs 2011;1.doi:10.4103/0974-6781.91283.

[80] Wang HV: A Unit Cell Approach for Lightweight Structure and Compliant Mechanism. Dep Mech Eng 2005;PhD:304.

[81] Murphy CM, O'Brien FJ: Understanding the effect of mean pore size on cell activity in collagen-glycosaminoglycan scaffolds. Cell Adhes Migr 2010;4:37781.doi:10.4161/cam.4.3.11747.

[82] O.HoffMAN: Strength of Orthotropic Materials 1962;1:200-6.

[83] Pal B, Gupta S, New a M: A numerical study of failure mechanisms in the cemented resurfaced femur: effects of interface characteristics and bone remodelling. Proc Inst Mech Eng H 2009;223:471-84.doi:10.1243/09544119JEIM488. 